\title{
RECENTES APLICAÇÕES SINTÉTICAS DE COMPOSTOS ORGÂNICOS TRICLORO(BROMO)METILA SUBSTITUÍDOS
}

Fabrício Gava Menezes, Hugo Gallardo e César Zucco*

Departamento de Química, Universidade Federal de Santa Catarina, CP 476, 88040-900 Florianópolis - SC, Brasil

Recebido em 1/7/10; aceito em 11/10/10; publicado na web em 16/11/10

RECENT SYNTHETIC APPLICATIONS OF TRICHLORO(BROMO)METHYL CONTAINING ORGANIC COMPOUNDS. This review presents the recent applications of some trihalomethyl carbinols, $\alpha, \alpha, \alpha$-trihalogenated carbonyl compounds and trichloro(bromo)methyl-containing heterocycles, acting as high versatile synthetic precursors in organic chemistry, based on the last 10 years' literature.

Keywords: trichloro(bromo)methyl compounds; synthetic applications.

\section{INTRODUÇÃO}

\section{O grupo tricloro(bromo)metila}

Compostos orgânicos contendo grupos $-\mathrm{CCl}_{3}$ ou $-\mathrm{CBr}_{3}$ em suas estruturas possuem diversas aplicações em diferentes áreas como bioquímica, agroquímica, produtos naturais, mas são reconhecidos, sobretudo, como reagentes altamente versáteis em síntese orgânica. O clorofórmio, por exemplo, o derivado mais simples contendo o grupo $-\mathrm{CCl}_{3}$, é um solvente de larga utilização em laboratórios. A Figura 1 mostra alguns compostos contendo o grupo $-\mathrm{CX}_{3}$, dotados de interessantes propriedades. O DDT, 1, é o inseticida mais conhecido da história. ${ }^{1} \mathrm{O}$ composto piridínico 2 é relatado como um agente antitumoral ${ }^{2}$ e o derivado arsênico $\mathbf{3}$ possui atividade antileucêmica. ${ }^{3} \mathrm{O}$ grupo $-\mathrm{CCl}_{3}$ é fundamental para a atividade biológica do Salubrinal, 4, uma molécula altamente eficaz na proteção de células sadias contra apoptose induzida por estresse excessivo e incorreto do retículo endoplasmático, fato responsável por importantes patologias como diabetes, Alzheimer e infecções virais. ${ }^{4,5}$ Com relação aos derivados contendo o grupo $-\mathrm{CBr}_{3}$, destaca-se o agente neurotóxico 5. ${ }^{6}$ Já o composto 6 é um dos componentes da alga vermelha Asparagopsis Taxiformis, que apresenta atividade contra aldose redutase em bovinos. ${ }^{7}$
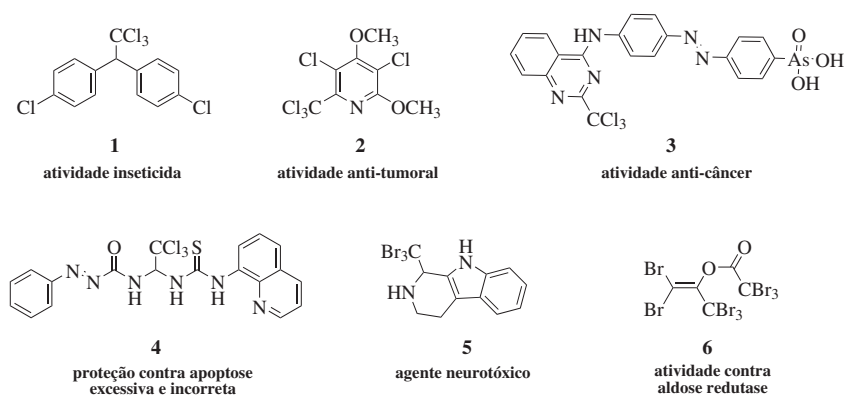

Figura 1. Alguns compostos importantes contendo $-\mathrm{CCl}_{3} \mathrm{Ou}-\mathrm{CBr}_{3}$

\section{Aplicações sintéticas de compostos orgânicos contendo - $\mathbf{C X}_{3}$}

O desenvolvimento de novas estratégias e metodologias em sín-

*e-mail: czucco@qmc.ufsc.br

\#Artigo em homenagem ao Prof. Hans Viertler tese orgânica é um acontecimento recorrente e promissor de pesquisa em Química, e, nesse contexto, compostos orgânicos contendo o grupo $-\mathrm{CX}_{3}(\mathrm{X}=\mathrm{Cl}, \mathrm{Br})$ têm sido citados na literatura como reagentes úteis e versáteis em diversas circuntâncias. Nesta revisão, serão apresentados exemplos relatados, principalmente na última década, envolvendo a utilização de tri-halometil carbinois (THMCs), compostos carbonilados $\alpha, \alpha, \alpha$-tri-halogenados (CCTHs) e heterociclos contendo $-\mathrm{CX}_{3}$ como precursores sintéticos de funções químicas variadas. Esses compostos atuam, muitas vezes, como peças fundamentais em estratégias de síntese de moléculas altamente elaboradas e de acentuada importância.

Em 1934, Fuson e Bull publicaram um artigo de revisão acerca da reação do halofórmio, apontando, já naquela época, utilização dessa reação em análises qualitativas e quantitativas, suas aplicações em síntese e explicações mecanísticas. ${ }^{8}$ De fato, a reação do halofórmio é uma das mais antigas na Química. Em 1822, Serulla, acidentalmente, verificou que o tratamento de etanol com hipoiodito, formado a partir de iodo em solução básica de hidróxido, levava à obtenção de iodofórmio e íon formato, por reação de oxidação do álcool a acetaldeído, seguida da tri-iodometilação e finalizada por hidrólise básica de $-\mathrm{CI}_{3}{ }^{8}$ Muitas aplicações e adaptações da reação de halofórmios são relatadas até hoje, algumas sendo objeto da presente revisão.

Os próprios halofórmios, por si sós, são interessantes precursores sintéticos, conforme exemplificado em duas publicações recentes. ${ }^{9,10}$ Dessa forma, o Esquema 1 apresenta a adição de bromofórmio a nitroalcenos, 7, na presença de magnésio, que, realizada a $0{ }^{\circ} \mathrm{C}$, forma os adutos de Michael, $\beta$-tribromometilnitroalcanos, 8 , com rendimentos de 56 a $95 \% .{ }^{9}$ Todavia, quando realizada sob refluxo ou com di-isopropilamideto de lítio (LDA), em vez de magnésio, a $-78{ }^{\circ} \mathrm{C}$, os produtos da reação são dibrometos vinílicos nitrados, 9, substratos bastante versáteis em síntese. Quando foram utilizados nitrocicloalcenos, os derivados cíclicos de $\mathbf{8}$ foram obtidos com diastereosseletividade de 75:25 a 100:0, sempre em favor do isômero trans. ${ }^{9}$

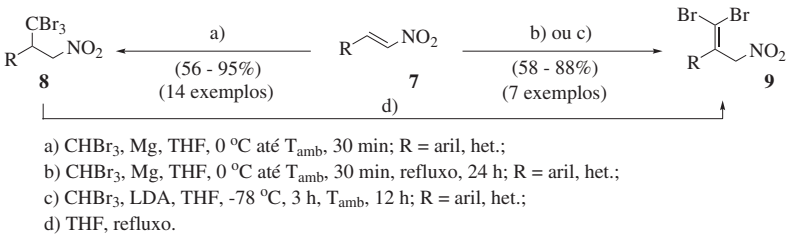

Esquema 1 
Outro exemplo recente envolve a condensação de $\alpha$-amino aldeídos quirais, 10, com $\mathrm{CHCl}_{3}$ e $\mathrm{CHBr}_{3}$, em presença de $\mathrm{CrCl}_{2}$ e $\mathrm{CrBr}_{2}$, respectivamente, levando à obtenção de aminas alílicas, importantes blocos de construção em síntese. ${ }^{10}$ Assim, uma série de $(S)$-alilaminas- $(E)$-clorovinílicas, 11, foi obtida com bons rendimentos (50 a 75\%) e excelente estereosseletividade, 90 a $98 \%$, em favor do isômero trans, sempre mantendo a configuração do aldeído de partida (Esquema 2). ${ }^{10}$

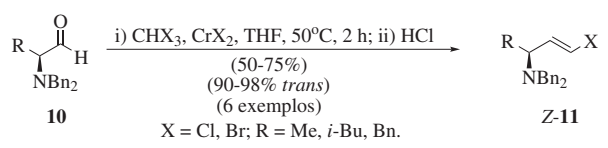

Esquema 2

Os halofórmios são, há tempo, conhecidos como fontes de dihalocarbenos ${ }^{11}$ e íons tri-halometilas.

\section{Tri-halometil carbinois - THMCs}

Existe um grande número de trabalhos relatando aplicações sintéticas de THMCs, sobretudo, dos derivados clorados. Os THMCs já foram revisados por Gukazyan e colaboradores, que publicaram, em 1991, métodos de preparação e algumas aplicações sintéticas. ${ }^{12}$ Os principais métodos de obtenção de THMCs envolvem reações de compostos carbonilados com íons tri-halometilas provenientes de diferentes fontes como halofórmios, ácido tri-haloacético e íon tri-haloacetato, tetracloreto de carbono, tri-haloacetaldeído, além de compostos organofosforados, estanhosos e de silício contendo o grupo $-\mathrm{CX}_{3}$. Os autores apontaram que o uso de THMCs se encontrava em estágio de desenvolvimento promissor, e, de fato, foi constatado, mais tarde, que as condições experimentais dos métodos de obtenção de THMCs foram aprimoradas e suas aplicações sintéticas cresceram consideravelmente.

Atualmente, os principais métodos de preparação de THMCs ainda envolvem as reações do ânion tri-halometila com espécies carboniladas, especialmente aldeídos, a partir de: $\mathrm{CHX}_{3}$, em meio básico, ${ }^{13-24}$ ácido tri-haloacético e tri-haloacetato, ${ }^{25-27}$ conforme o Esquema 3.

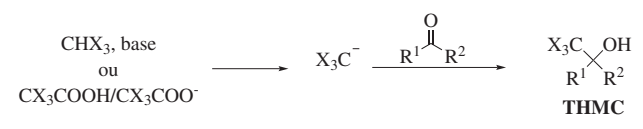

Esquema 3

Todavia, outros métodos são relatados na literatura e envolvem as reações de cloral e/ou bromal com alcenos, ${ }^{28}$ alcinos, ${ }^{29}$ enolatos,,${ }^{30,31}$ iminas $^{32}$ e por redução de tri-halometilcetonas. ${ }^{13,15,24,33-35}$ Esse último método chama a atenção pelo fato de diferentes catalisadores serem utilizados com o objetivo de realizar reações estereosseletivas para obtenção de THMCs enantiomericamente enriquecidos, conforme exemplificado no Esquema 4. Em artigo de revisão, Corey e colaboradores exploraram as oxazaborolidinas em reduções de cetonas, incluindo derivados contendo grupos tri-halometila. ${ }^{29}$ Essas cetonas halogenadas pró-quirais podem ser reduzidas enantiosseletivamente ao THMC 12, com configuração absoluta $R$, quando na presença do catalisador 13. ${ }^{15}$ Em outro exemplo, Ramachandran e colaboradores relataram a obtenção dos THMCs 14, com configuração absoluta $S$, mediada pelo catalisador $\mathbf{1 5}$. $^{35}$

Assim, a partir dos métodos relatados, vários THMCs podem ser preparados e, em seguida, utilizados como precursores sintéticos para obtenção de diversas funções químicas, das quais, algumas serão comentadas a seguir.

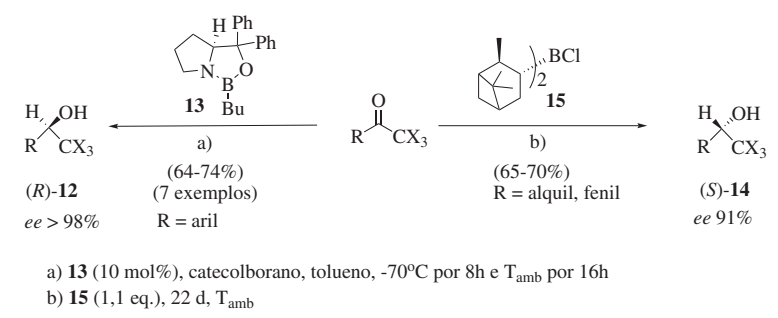

Esquema 4

\section{Obtenção de $\alpha$-aminoácidos e outros ácidos carboxílicos $\alpha$-substituídos}

Há mais de um século foi apresentada a reação de Jocic, ${ }^{36}$ que diz respeito à obtenção de ácidos carboxílicos $\alpha$-substituídos a partir da reação de THMCs com diferentes nucleófilos, em solução aquosa de $\mathrm{NaOH}$. O mecanismo envolve a formação do dicloroepóxido 16 pela reação de THMCs com $\mathrm{HO}^{-}$, seguida da abertura do anel pelo ataque nucleofílico tipo $\mathrm{S}_{\mathrm{N}} 2$, com inversão de configuração, para formar o cloreto de ácido $\alpha$-substituído 17 , que é hidrolisado ao respectivo ácido carboxílico 18 (Esquema 5). ${ }^{37}$

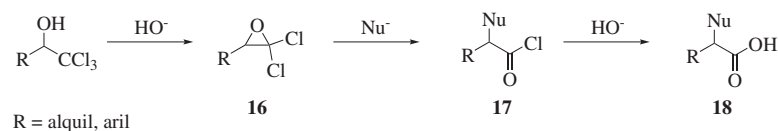

$$
\begin{aligned}
& \mathrm{Nu}=\mathrm{R}^{1} \mathrm{O}, \mathrm{HO}, \mathrm{R}^{1} \mathrm{~S}, \mathrm{R}^{1}{ }_{2} \mathrm{~N}, \mathrm{~N}_{3}
\end{aligned}
$$

Esquema 5

Uma aplicação do método de Jocic foi documentada por Corey e Link, relativamente à síntese enantiosseletiva de $\alpha$-aminoácidos, ${ }^{13,29}$ conforme o Esquema 6. Por exemplo, os $(R)$-THMCs 19, obtidos a partir de redução assimétrica de tri-halometilcetonas são transformados nos dicloroepóxidos 20, que sofrem abertura nucleofílica pelo ataque tipo $\mathrm{S}_{\mathrm{N}} 2$ do íon azida, com consequente inversão de configuração, para formar 21, com configuração absoluta $S$. Estes sofrem hidrólise para formar 22, que, por sua vez, são reduzidos por hidrogenação catalítica aos $(S)$ - $\alpha$-aminoácidos $\mathbf{2 3}$, obtidos com $88-98 \%$ de rendimento.

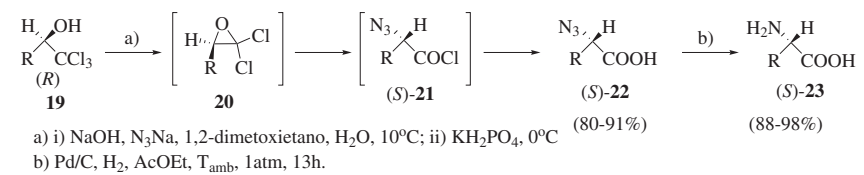
b) $\mathrm{Pd} / \mathrm{C}, \mathrm{H}_{2}$, AcOEt, $\mathrm{T}_{\mathrm{amb}}, 1 \mathrm{~atm}, 13 \mathrm{~h}$.
$\mathrm{R}=$ alqui, benzonaftil.

\section{Esquema 6}

Variações do método de Corey-Link foram apresentadas no intuito de aumentar a enantiosseletividade dos $\alpha$-aminoácidos, como na metodologia de Aitken e colaboradores, usada para a obtenção de $(S)$-arilglicinas, com excesso enantiomérico $\geq 97 \%$, a partir do (R)-THMC, utilizando DBU em vez de $\mathrm{NaOH} .{ }^{15}$ De fato, os métodos de Jocic e Corey-Link lançaram uma excelente alternativa para as sínteses de derivados de ácidos carboxílicos $\alpha$-substituídos, a partir do que interessantes trabalhos foram publicados.

\section{Obtenção de di-haletos vinílicos e Z-cloro-vinil-olefinas}

Da mesma forma que $\mathrm{CHBr}_{3}$ (Esquema 1), ${ }^{9}$ THMCs também são explorados na obtenção de di-haletos vinílicos que, ${ }^{38,39}$ como importantes intermediários sintéticos, têm atraído a atenção, sobretudo, por serem estratégicos na síntese de compostos dotados de atividade antiHIV. ${ }^{38}$ A transformação de THMCs em di-haletos vinílicos mediada por índio metálico não é efetiva, uma vez que diclorometil cabinois 
são formados como subprodutos. Todavia, quando os respectivos THMCs são protegidos nas formas de acetato, mesilato ou tosilato, nas mesmas condições experimentais, tricloro- e tribromometil carbinois produzem, de forma limpa e exclusiva, os respectivos derivados olefínicos di-halogenados 24, cujo rendimento foi de 60 a 97\% (Esquema 7). ${ }^{39}$ Quando em lugar de índio metálico as reações são mediadas por $\mathrm{SmI}_{2},{ }^{38}$ tanto os THMCs desprotegidos quanto os respectivos acetatos sofrem eliminação redutiva para formar derivados de $\mathbf{2 4}$ entre 70 a $96 \%$ de rendimento, em tempos de reação muito menores. Em ambos os métodos, os derivados carbinólicos aromáticos e heteroaromáticos apresentaram resultados melhores que os alifáticos.,39

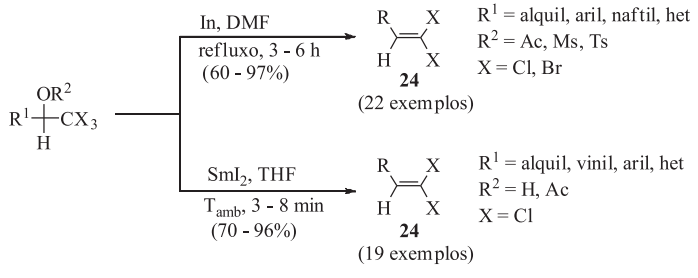

Esquema 7

Outro exemplo envolvendo a preparação de di-haletos vinílicos, com rendimentos moderados, pode ser destacado. Trata-se da reação de bromal com 1,4-dimetoxibenzeno, em meio ácido, para formar o THMC 25 que, sob $\beta$-eliminação em meio básico (metóxido de sódio em metanol), produz o di-haleto vinílico $\mathbf{2 6}$, com $33 \%$ de rendimento global (duas etapas), conforme o Esquema $8 .^{40}$ Estruturalmente, esses compostos podem ser considerados interessantes blocos de construção altamente conjugados.

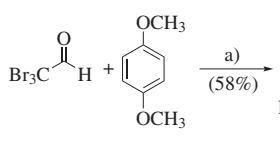

a) $\mathrm{AcOH}, \mathrm{H}_{2} \mathrm{SO}_{4}, \mathrm{~T}$ b) $\mathrm{CH}_{3} \mathrm{ONa}, \mathrm{CH}_{3} \mathrm{OH}$, refluxo

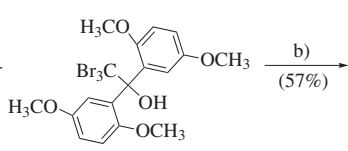

25

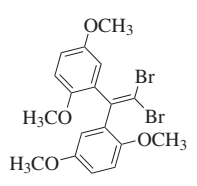

26

\section{Esquema 8}

Di-haletos vinílicos podem ser também preparados em reações mediadas por eletroquímica, como será visto mais a adiante..$^{32,33}$

Os THMC podem ser utilizados, em sua forma livre ou $O$ substituídos (protegidos) nas sínteses estereosseletivas de (Z)-halovinil olefinas substituídas, conforme apresentado no Esquema 9. Dessa forma, alcoóis alílicos-(Z)-cloro-olefínicos, 27, e (Z)-cloretos vinílicos, 28, são obtidos quando THMCs são tratados com $\mathrm{CrCl}_{2}$, seguido de reação com diferentes aldeídos ou tratamento com água, respectivamente. ${ }^{41}$ Já quando os THMCs são $O$-acilados, reagem $\operatorname{com~} \mathrm{CrCl}_{2}$ para formar os derivados alcoxicloro olefínicos $\mathbf{2 9}$, e, nas mesmas condições, os derivados $O$-alquil e $O$-silil levam à formação dos derivados 30 ${ }^{42} \mathrm{Em}$ todos os casos, os produtos foram obtidos com estereoquímica $Z$ e rendimentos entre 61 e $98 \%$.

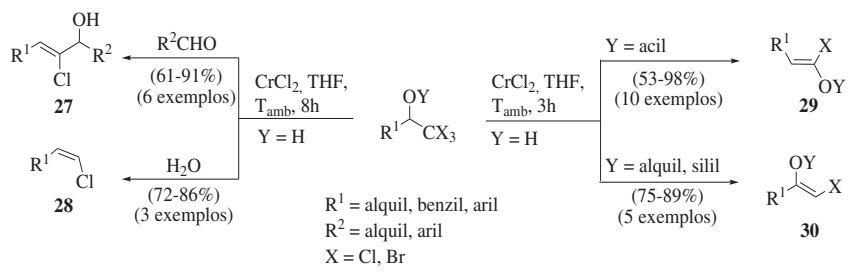

Esquema 9

Ainda, 1-alcoxi-2-cloroetenos com geometria $Z$ podem ser obtidos como produtos majoritários a partir de 2,2,2-tricloroacetatos de alquila tratados com uma mistura de $\mathrm{CuCl}$ e bipiridina. ${ }^{43}$

\section{Transformação de THMC em acetilenos terminais}

A tosilação ou mesilação de THMCs leva aos produtos $\mathbf{3 1} \mathrm{com}$ altos rendimentos (81 a 98\%). Esses produtos reagem, em seguida, com excesso de iodeto de metila para formar os acetilenos terminais 32 (Esquema 10), por um processo in situ que envolve formação de dicloreto vinílico, eliminação de $\mathrm{HCl}$, litiação e protonação. ${ }^{17,26}$ Essa metodologia foi eficaz para escalas até $400 \mathrm{~g}$, podendo ser aplicada para obtenção de um intermediário chave na síntese do Efavirenz, um fármaco dotado de atividade anti-HIV. ${ }^{17}$ Os acetilenos terminais são reagentes importantes para a formação de novas ligações $\mathrm{C}-\mathrm{C}$, quando reagem com um haleto vinílico ou arílico. Conhecida como reação de acoplamento cruzado catalisado por $\mathrm{Pd}-\mathrm{Cu}$, ou simplesmente como acoplamento de Sonogashira, ${ }^{44-48}$ a reação ocorre em condições suaves para uma grande variedade de substratos, produzindo compostos acetilênicos dissubstituídos. Essa reação tem sido amplamente usada na síntese de produtos naturais e materiais avançados, como dendrímeros, ${ }^{49}$ cristais líquidos luminescentes, ${ }^{50-52}$ nanomáquinas ${ }^{53} \mathrm{e}$ compostos emissores para OLEDs. ${ }^{54,55}$

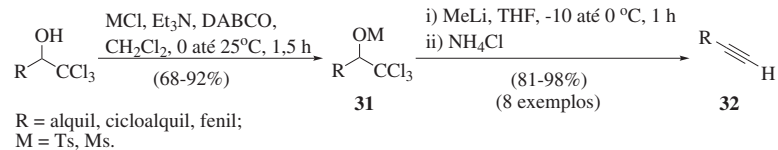

Esquema 10

Os acetilenos terminais são, também, importantes reagentes nas reações de click chemistry, com ampla utilização na síntese de compostos orgânicos com interesse principalmente biológico, por fornecer uma diversidade química conveniente para uma varredura biológica e por constituir-se em um protocolo de síntese rápido e eficiente. ${ }^{56-59}$ A partir da reação de click entre um aril acetileno terminal e uma azida obtém-se o heterociclo 1,2,3-triazol-1,4-dissubstituído com $100 \%$ de regiosseletividade.$^{60,61}$ Os triazois, em geral, têm atraído a atenção das indústrias farmacêuticas para a preparação de fármacos ${ }^{62}$ devido à elevada estabilidade e por não apresentarem toxicidade. Atualmente, encontram também aplicações nas áreas tecnológicas de cristais líquidos ${ }^{63}$ e de dispositivos emissores de luz. ${ }^{64}$

\section{THMC mediando reações de homologação}

Dentre os poucos métodos que existem para a homologação, do tipo one pot, de aldeídos a ácidos carboxílicos, o de Jocic é uma excelente alternativa, conforme relatado por Snowder e Cafiero. ${ }^{25}$ Nessas reações, os aldeídos são transformados em THMCs que, tratados seguidamente com $\mathrm{NaBH}_{4}$ ou $\mathrm{Na}\left[\mathrm{PhSeB}(\mathrm{OEt})_{3}\right]$, em meio alcoólico alcalino, conduzem a diferentes ácidos carboxílicos com rendimentos de 76 a 95\% (Esquema 11). Incluem-se, nessa alternativa, aldeídos quirais que resultaram em produtos com retenção de configuração.

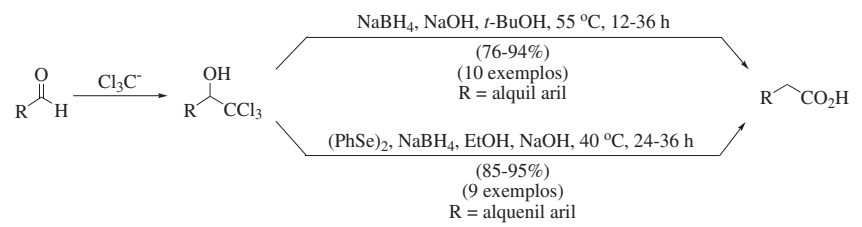

Esquema 11

Como outros alcoóis secundários, THMCs podem ser oxidados a cetonas sob diferentes condições. Todavia, existem outras formas 
de obtenção de cetonas a partir de THMCs. Dessa forma, as reações de THMCs terciários cíclicos 35, com diferentes aldeídos, mediadas por $\mathrm{CrCl}_{2}$, levam à formação das cetonas $\alpha, \beta$-insaturadas $36 \mathrm{com}$ expansão do anel (homologação). ${ }^{65}$ Essas reações foram consideradas eficazes tanto para derivados clorados quanto bromados, embora apresentassem rendimentos variados (11 a 82\%) dependendo da estrutura do reagente de partida (Esquema 12). Outro exemplo de obtenção de cetonas envolve THMCs alílicos, vinílicos, aromáticos e heterocicloss nas sínteses regioespecíficas de $\alpha$-cloro cetonas, a partir de reações com $\mathrm{CuCl}$ e bipiridina, em éter metílico, sob refluxo, com rendimetos de 84 a $96 \% .{ }^{66}$

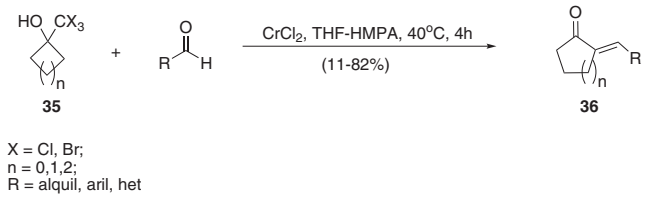

Esquema 12

\section{THMCs na preparação de heterociclos}

THMC são usados, também, como precursores sintéticos para obtenção de heterociclos, alguns polifuncionalizados, demonstrados a seguir para derivados de quinolina, tiazolidinona e furano. Assim, a reação de mesilação do triclorometil-alquinol $\mathbf{3 4}$ forma o derivado protegido 35 (as reações com o análogo bromado e/ou anidrido trifluormetanossulfônico também formam os respectivos produtos) que, em seguida, sofre adição nucleofílica, na ligação tripla, por aminas para formar as $\alpha, \alpha$-dicloroetilenaminonas 36, com rendimentos entre 37 e $90 \%$. Essas enonas são conhecidas por atuarem como intermediários-chave em sínteses de diferentes heterociclos. Quando derivados de $\mathbf{3 6}$ foram aquecidos em ácido polifosfórico (PPA) foram obtidos, por ciclização intramolecular, os derivados 2-fenil-4-diclorometilquinolinas $\mathbf{3 7}$ em excelentes rendimentos, de 92 a $96 \%$ (Esquema 13). ${ }^{31}$

$$
\begin{aligned}
& 34 \\
& \mathrm{R}^{1}=\mathrm{H}, \mathrm{Me} \text {; } \\
& \mathrm{R}^{2}=\text { alquil, alil, aril; }
\end{aligned}
$$

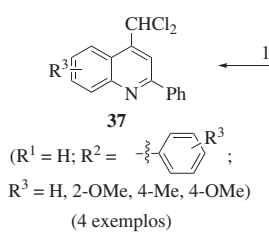

Esquema 13

A alilação de THMC com diferentes brometos alílicos leva à formação de triclorometil éteres $\mathbf{3 8}$, que, por sua vez, sofrem ciclização intramolecular por transferência de átomo (HATRC), mediada por $\mathrm{CuCl} /$ bipiridina, culminando no intermediário diclorotetrahidrofuranoico 39. Esses compostos sofrem dupla eliminação de $\mathrm{HCl}$ em meio básico para formar 40, que sofrem isomerização da ligação dupla, originando os derivados clorofuranoicos 41. ${ }^{67}$ Dentre as aplicações sintéticas dos derivados $\mathbf{4 1}$, destaca-se seu uso como substratos apropriados às reações de ciclo adição de Diels-Alder. Dessa forma, a cicloadição [4+2] com acetileno ativado 42 leva ao intermediário 43, que sofre abertura de anel, seguida de aromatização, para formar os derivados benzênicos hexassubstituídos $44 .{ }^{67} \mathrm{De}$ modo geral, os rendimentos foram de moderados para bons em todas as etapas (Esquema 14).

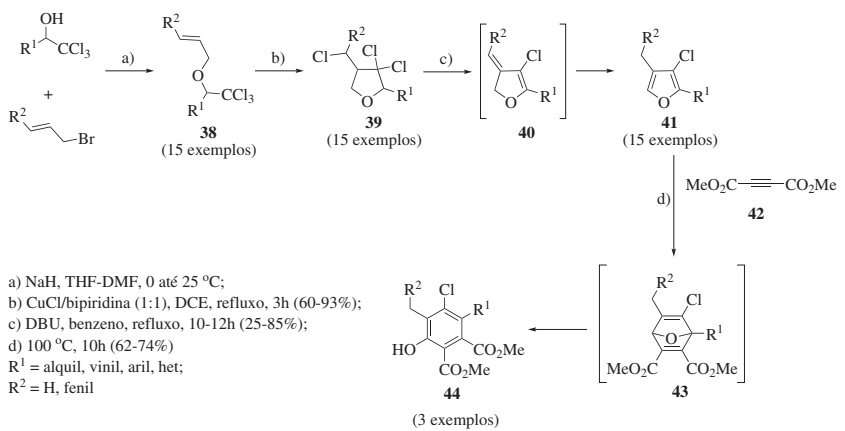

Esquema 14

Guirado e colaboradores relataram interessantes trabalhos envolvendo compostos orgânicos contendo $-\mathrm{CX}_{3}$ em reações mediadas eletroquimicamente. ${ }^{32,33} \mathrm{Em}$ um dos exemplos, as reações aldólicas de acetofenonas substituídas com cloral produziram os THMCs 45 que, desidratados, geram derivados 46, os quais são convertidos eletroquimicamente em dicloretos vinílicos $\mathbf{4 7}$. Esses são isomerizados aos derivados contendo o grupo diclorometila $\mathbf{4 8}$ e reagem com hidrazinas para formar derivados 3-aril-5-diclorometil-2-pirazolinas 49, com rendimentos acima de $75 \%$ em todas as etapas da rota sintética. $\mathrm{O}$ Esquema 15 apresenta o método para obtenção desses heterociclos, que são conhecidos por suas importantes atividades biológicas e como precursores sintéticos. ${ }^{32,33}$

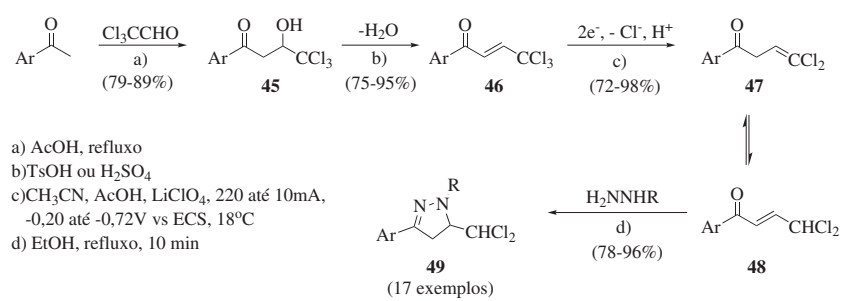

\section{Esquema 15}

As reações de THMCs alquílicos, alílicos e aromáticos com tioureias, em meio básico, levam à formação de 2-imino-4-tiazolidinonas, com rendimentos de 47 a $81 \%$, a partir de uma adaptação do método de Reeve. ${ }^{21}$ Esses heterociclos, com conhecidas aplicações biológicas, podem ser preparados por um método one-pot envolvendo três componentes: aldeídos, clorofórmio e tioureia, com $\mathrm{DBU}, \mathrm{NaOH}$ aquoso e DME, com rendimentos de 25 a $46 \%{ }^{21}$

\section{COMPOSTOS CARBONILADOS $\alpha, \alpha, \alpha$-TRI- HALOGENADOS (CCTHS)}

Quando o grupo $-\mathrm{CX}_{3}$ está ligado diretamente a um composto carbonilado, abre-se um novo leque de possibilidades sintéticas. $\mathrm{O}$ grupo $-\mathrm{CX}_{3}$ é bastante estável em diversas condições, possibilitando que reações típicas de compostos carbonilados (redução, adição nucleofílica, substituição nucleofílica) possam ocorrer, mantendo a integridade do grupo $-\mathrm{CX}_{3}$. Todavia, há casos em que o grupo $-\mathrm{CX}_{3}$ pode ser substituído ou mesmo decomposto, acarretando transformações indesejáveis.

\section{-CX $\mathrm{C}_{3}$ atuando como grupo de saída}

O grupo $-\mathrm{CX}_{3}$ é comumente aceito como grupo de saída, especialmente quando parte integrante de derivados cetônicos. ${ }^{8}$ Estudos relatam que esses reagentes podem ser hidrolisados a ácidos carboxílicos ${ }^{68,69}$ ou atuarem como agentes acilantes e benzoilantes de 
alcoóis, ${ }^{70-75}$ aminas, ${ }^{76-80}$ hidrazinas ${ }^{70}$ e nucleófilos de carbono,${ }^{70}$ sempre em condições brandas, conforme o Esquema 16.

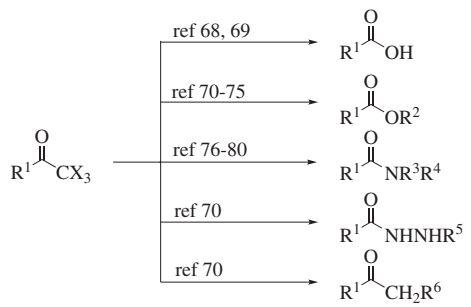

Esquema 16

Uma classe interessante de cetonas $\alpha, \alpha, \alpha$-tri-halogenadas são as hexacloroacetona (HCA) e hexabromoacetona (HBA). As reações dessas cetonas com alcoóis, ${ }^{81,83}$ aminas, ${ }^{82,83}$ e diaminas, ${ }^{83-85}$ conduzem à substituição do grupo $-\mathrm{CX}_{3}$ simultaneamente à tri-haloacetilação do nucleófilo. Esse comportamento é mais efetivo para a espécie clorada devido, sobretudo, ao menor impedimento estérico do $-\mathrm{CCl}_{3}$ em comparação com $-\mathrm{CBr}_{3}{ }^{86,87}$

Um exemplo relatado por Nishikawa e colaboradores, envolvendo a substituição nucleofílica de $-\mathrm{CCl}_{3}$, aponta a transformação one-pot de diferentes tricloroacetamidas em carbamatos, por um intermediário isocianato. ${ }^{88} \mathrm{O}$ tratamento da tricloroacetamida com uma solução de $\mathrm{Na}_{2} \mathrm{CO}_{3}$, em DMF, sob refluxo, forma derivados isocianatos, que são capturados, in situ, por alcoóis, na presença de $n-\mathrm{Bu}_{4} \mathrm{NCl}$ e $\mathrm{CuCl}$, para formar carbamatos, com rendimentos de moderados para ótimos. No Esquema 17 está apresentada a transformação da tricloroacetamida 50 no intermediário 51, que é capturado, in situ, por álcool benzílico para formar o carbamato $\mathbf{5 2} \mathrm{com} 96 \%$ de rendimento.

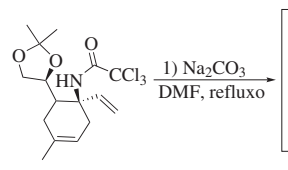

50

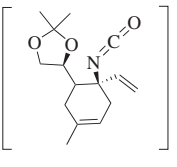

51

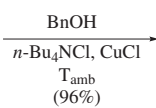

$(96 \%)$

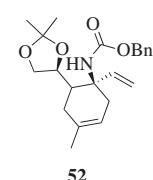

Esquema 17

\section{O uso de CCTH como agentes halogenantes}

Observando-se as estruturas químicas dos compostos orgânicos contendo $-\mathrm{CX}_{3}$, pode-se associar esses grupos a boas fontes de halogênio ou a bons agentes halogenantes, conforme várias citações da literatura.

\section{Obtenção de haletos de alquila e de acila}

Certamente, uma das aplicações mais importantes e amplamente utilizada dos CCTHs é a capacidade de mediar conversões de alcoóis a haletos de alquila e de ácidos carboxílicos a haletos de acila e benzoíla, com $\mathrm{PPh}_{3}$. Essa reação é uma variação da reação de Mitsunobu, ${ }^{89,90}$ conforme apresentado no Esquema 18. O ataque da trifenilfosfina a um átomo de halogênio de um CCTH leva à formação da espécie $\mathbf{5 3}$ que, na presença de alcoóis ou de ácidos carboxílicos, representados por $\mathbf{5 4}$, formará o sal $\mathbf{5 5}$, juntamente com a $\mathrm{CHX}_{2} \mathrm{COR}^{1}$, propiciando o ataque nucleofílico do íon cloreto ao carbono adjacente ao fósforo, conforme representado por 56, levando à formação dos haletos de alquila, acila ou benzoila, representados por $\mathbf{5 7}$, além de óxido de trifenilfosfina, subproduto da reação.

Diversos CCTHs têm sido usados como agentes halogenantes, ${ }^{82,89-94}$ em diferentes formas (ácidos carboxílicos, ésteres, amidas, aldeídos e cetonas, todos tri-halogenados). De acordo com

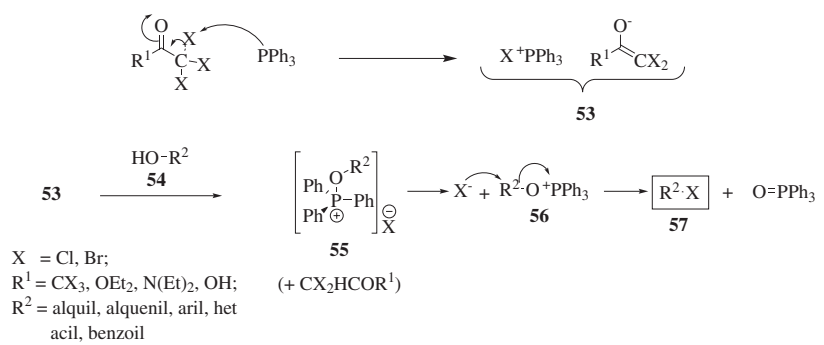

Esquema 18

estudos de reatividade de Chavasiri e colaboradores, ${ }^{91}$ o poder de halogenação desses agentes pode ser diretamente relacionado ao efeito retirador de elétrons do outro substituinte ligado à carbonila, podendo-se cuidadosamente estabelecer HCA, HBA e tricloroacetonitrila como os reagentes mais efetivos. Esse método de halogenação foi aplicado em diversos trabalhos, ${ }^{95,96} \mathrm{com}$ destaque para as conversões, in situ, de haletos de acila e benzoíla em amidas ${ }^{82,92,95} \mathrm{e}$ cetonas, ${ }^{91}$ a partir das reações com aminas e nucleófilos de carbono, respectivamente.

Segundo o Esquema 19, a oxidação de fosfinas e óxidos de fosfinas desempenha papel crucial no mecanismo de halogenação. Há exemplos na literatura em que a obtenção do haleto de alquila não é objetivo principal da síntese. Bergin e colaboradores publicaram a inédita oxidação de racematos de fosfinas por um sistema contendo álcool quiral (especialmente derivados terpênicos disponíveis comercialmente a preços acessíveis) e HCA, levando aos respectivos óxidos de fosfinas com rendimentos acima de $95 \%$ e, em alguns casos, excesso enantionético de até $80 \% .{ }^{97}$ Compostos fosforilados quirais possuem diferentes aplicações em catálise assimétrica, e, em geral, o centro estereogênico encontra-se em um átomo de carbono. Todavia, nesses exemplos a quiralidade está no átomo de fósforo. No trabalho de Bergin, foi apresentado um exemplo notável de síntese em duas etapas: inicialmente, a fosfina $\mathbf{6 0}$ foi oxidada ao óxido de fosfina $\mathbf{6 1}$ com ótimo rendimento e bom excesso enantiomérico (95 e $80 \%$, respectivamente), por meio da reação com (-)-mentol/HCA, em tolueno, para ser dimerizada a bifosfina 62, que foi obtida com bom rendimento e excelente excesso enantiomérico $(99,9 \%)$, após recristalização (Esquema 19). ${ }^{97}$

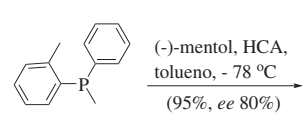

60

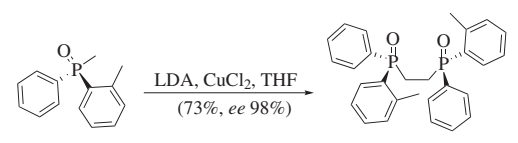

$61(R)$

$62(R, R)$

\section{Outras halogenações mediadas por CCTHs}

Outras halogenações com compostos contendo $-\mathrm{CX}_{3}$, como, por exemplo, organosfosforados, incluindo reações estereosseletivas, ${ }^{98,99}$ enaminas ${ }^{100} \mathrm{e}$ anéis aromáticos são encontradas na literatura. ${ }^{101}$ Recentemente foi mostrado que a aziridina $N$-tosilada 63, derivada do cicloexano, reage com CCTHs (HCA, ácido tricloroacético ou tricloroacetamida), em $\mathrm{PPh}_{3}$, para formar a $\alpha$-cloroamina $N$-tosilada 64, por abertura do anel aziridínico, com ótimos rendimentos (87 a 97\%) em condições brandas (Esquema 20). ${ }^{102,103}$

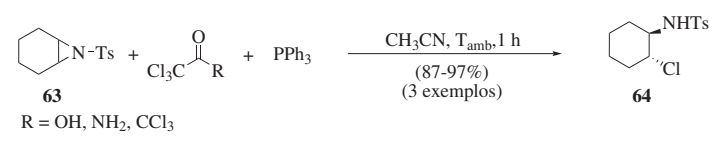

Esquema 20 


\section{Rearranjo de Overman}

No Esquema 17 foram realizadas reações partindo-se de tricloroacetamidas alílicas bem elaboradas, obtidas, muitas vezes, por um rearranjo [3,3]-sigmatrópico (aza-Claisen). Essa reação, conhecida como rearranjo de Overman, envolve a adição nucleofílica de um álcool alílico 65 à tricloroacetonitrila para formar tricloroacetoimidato 66, que sofre um rearranjo pericíclico para formar uma tricloroacetamida, 67 (Esquema 21). ${ }^{104-108}$ Esse rearranjo tem sido objeto central de diversos trabalhos de síntese orgânica, especialmente, envolvendo a obtenção de produtos naturais nitrogenados. ${ }^{104}$ Algumas virtudes do rearranjo de Overman são as suaves condições empregadas nas reações, a transferência de quiralidade de alcoóis quirais e a enantiosseletividade a partir de misturas racêmicas utilizando-se catalisadores quirais.

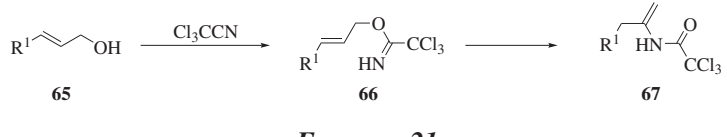

$$
\begin{aligned}
& \text { Esquema } 21
\end{aligned}
$$

Sínteses estereosseletivas de compostos carbonilados $\alpha$-halogenado- $\alpha, \beta$-insaturados

Recentemente, considerável atenção tem sido prestada às reações de condensação estereosseletiva de CCTHs com espécies carboniladas, especialmente aldeídos e cetonas, mediadas por diferentes metais. Essas reações levam à formação de derivados carbonilados $\alpha$-halogenados- $\alpha, \beta$-insaturados, que podem atuar como importantes blocos de construção para as sínteses de aminoácidos, heterociclos, aziridinas, polímeros, além de fármacos e produtos naturais. ${ }^{109,110}$ Falck e colaboradores relataram uma série de trabalhos explorando essas reações. ${ }^{109-112} \mathrm{O}$ Esquema 22 mostra as condensações de tri-haloacetatos de alquila com aldeídos que, com um equivalente de $\mathrm{Cr}(\mathrm{II})$, formam os intermediários $\mathbf{6 8}$ que, na presença de outro equivalente de $\mathrm{Cr}$ (II), dão origem aos (Z)- $\alpha$-haloacrilatos 69, com rendimentos de 91 a $100 \%$ e alta seletividade em favor do isômero $Z$ (>99\%). Controlando-se as condições experimentais, foram isoladas, também, a partir de 68, pela adição de água, as di-haloidrinas 70, porém, com rendimentos mais modestos. ${ }^{109}$

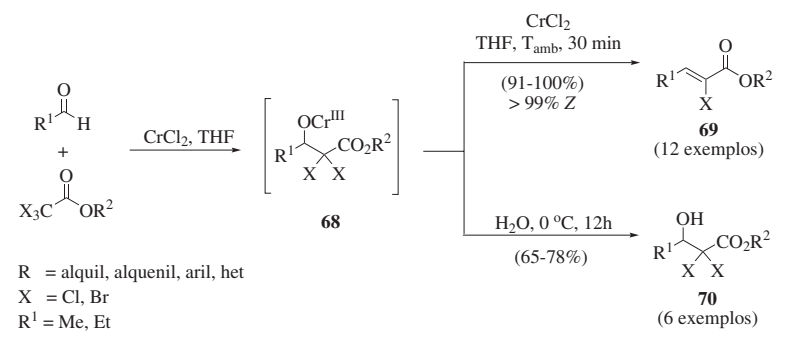

Esquema 22

Como o método se mostrou bastante interessante, outros reagentes foram testados, e, dessa forma, as condensações de aldeídos com triclorocetonas e tricloroacetamidas, mediadas por $\mathrm{Cr}(\mathrm{II})$, levaram à obtenção de (Z)- $\alpha$-cloro-cetonas e ( $Z$ )- $\alpha$-cloro-amidas, ambas $\alpha, \beta$ insaturadas, com rendimentos de $89-98 \%$, e estereosseletividade $Z>$ $99 \%$, além de dicloroidirinas com rendimentos de $75-87 \%$, quando as quantidades de reagentes e temperatura foram controladas. ${ }^{11}$ Apesar dos ótimos resultados, a toxicidade do cromo é um fator negativo nessas reações, e, por isso, foi desenvolvida uma metodologia visando ao uso de $\mathrm{Fe}(0)$ como mediador dessas condensações, que se mostrou efetiva tanto em termos de rendimento quanto de estereosseletividade dos (Z)- $\alpha$-cloro-acrilatos 71 (Esquema 23). ${ }^{112}$ No mesmo trabalho foram estudas e comparadas as condensações de cetonas com tricloroacetatos de metila, mediadas por $\mathrm{Cr}$ (II) e $\mathrm{Fe}(0)$, verificando-se, para ambos os metais, que a estereosseletividade foi em favor do isômero $E$ das $\alpha$-cloro-cetonas- $\alpha, \beta$-insaturadas 72, ou seja, inversa àquela envolvendo condensações com aldeídos. Por meio desses resultados, foi verificado que tanto os rendimentos quanto a estereosseletividade são dependentes das estruturas dos reagentes, e, de modo geral, os resultados das reações mediadas por $\mathrm{Fe}(0)$ foram superiores aos das reações com Cr(II), especialmente nas condensações envolvendo cetonas. Conforme verificado no Esquema 23, as reações com aldeídos apresentaram estereosseletividades maiores que com cetonas, chegando a $>99 \%$.

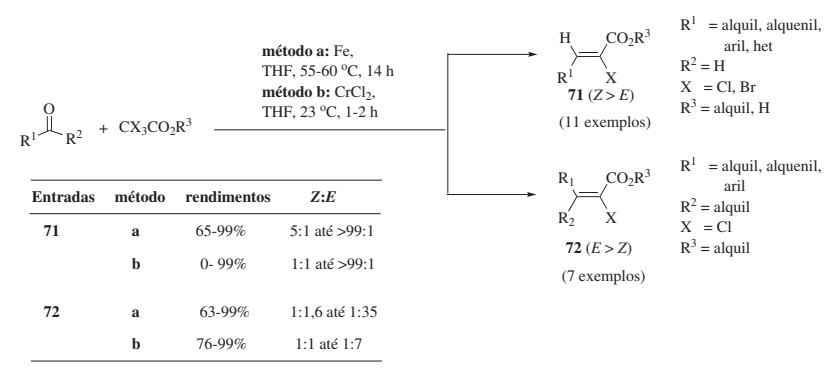

Esquema 23

Concellón e colaboradores usaram manganês como mediador das condensações de aldeídos com acetatos de alquila e $\mathrm{N}$-alquilacetamidas, ambos $\alpha, \alpha, \alpha$-triclorados, produzindo $(Z)$ - $\alpha$-cloro-acrilatos e (Z)- $\alpha$-cloro-acrilamidas, com estereossletividade $>98 \%$ em favor do isômero $Z$ e rendimentos de $62-95 \%$ e $73-88 \%$, respectivamente. ${ }^{113,114}$ Com alguns desses produtos obtidos, foram realizadas reações de substituição e redução na carbonila, verificando-se que tanto o cloro quanto a dupla ligação permaneceram intactas. ${ }^{113,114}$

Nesses trabalhos ${ }^{109-114}$ foram utilizados aldeídos e cetonas funcionalizadas, contendo grupos olefínicos, anilínicos, anisólicos, nitro, heterociclos, dentre outros, e, mesmo assim, as reações ocorreram exclusivamente na carbonila, o que torna o método excelente alternativa à obtenção de blocos de construção polifuncionalizados.

Em outro exemplo, Esquema 24, apresenta-se a condensação de aldeídos com a trifluortribromoacetona, 73 , mediada por $\mathrm{Ti}(\mathrm{O}-i \text {-Pr })_{4}$ e $\mathrm{Ph}_{3} \mathrm{P}$, em uma etapa, levando à obtenção de blocos de construção do tipo alcoóis bromoalílicos trifluormetilados $\mathbf{7 4}$, com rendimentos de 54 a $88 \%$ e excepcional estereosseletividade (100:0) em favor do isômero $Z$. $^{115}$

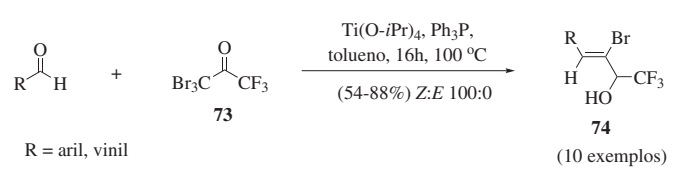

Esquema 24

\section{CCTHs na obtenção de heterociclos}

O uso de CCTHs como precursores sintéticos para obtenção de heterociclos está bem documentado na literatura. Snapper e colaboradores sintetizaram uma série de lactamas bicíclicas polifuncionalizadas mediadas pelo catalisador de rutênio Grubb 1, representado por 75, conforme Esquema 25. ${ }^{116}$ As reações foram divididas em dois tipos: intramolecular e combinação de intra e intermolecular, ambas sequenciais e em one pot, com formação de múltiplas ligações carbono-carbono, por processos simultâneos de RCM (ring closing metathesis) e adição de Kharasch. Dessa forma, a ciclização 
intramolecular de dienos derivados de tri-halocetamidas $\mathbf{7 6}$ leva à formação das lactamas bicíclicas 77 tri-halogenadas, incluindo derivados $N$-tosilados e $N$-benzilados, com rendimentos de 55 a $89 \%$, pela formação simultânea de duas novas ligações carbono-carbono. Quando na presença de estireno, essas reações produzem os derivados 78, com diastereosseletividade de 1:1, formados a partir de três novas ligações carbono-carbono e duas ligações $\mathrm{C}-\mathrm{Cl}$. Outro método foi relatado para formação de $\alpha, \alpha$-diclorolactonas a partir da ciclização intramolecular de tricloroacetamidas $N$-alílicas, em 1,4-dimetilpiperazina, por radical livre. Esse método pode ser aplicado como parte do procedimento da síntese total de alcaloides. ${ }^{117}$
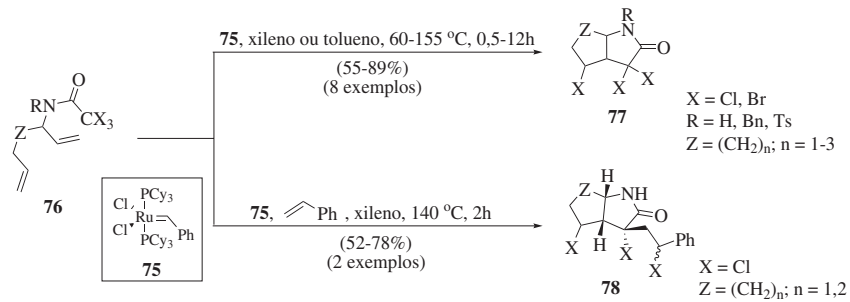

Esquema 25

Os ésteres $\alpha, \alpha, \alpha$-tri-halogenados, também CCTHs, foram usados para a síntese de heterociclos. Em outro exemplo, Sanpper usou o catalisador $\mathbf{7 5}$ na reação do tricloroacetato de etila com o estireno para obter o aduto triclorado $\mathbf{7 9}$, com rendimento moderado, e que pode ser ciclizado, com HCl, à lactona $\alpha, \alpha$-diclorada 80 (Esquema 26). ${ }^{118}$ Os rendimentos para $\mathbf{7 9}$ e $\mathbf{8 0}$ foram de 57 e $89 \%$, respectivamente.

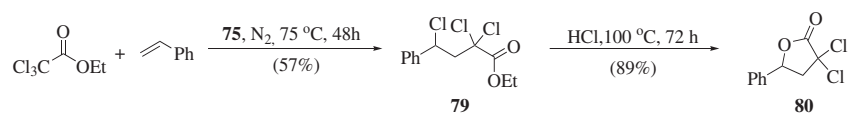

Esquema 26

Barba e colaboradores obtiveram cumarinas a partir de ésteres triclorados, por mediação eletroquímica, conforme Esquema 27. ${ }^{119,120}$ Assim, a reação de 2-hidroxifenonas com cloreto de tricloroacetila leva aos ésteres $\mathbf{8 1}$ que são convertidos nas cumarinas $\mathbf{8 2}$, com 33 a $77 \%$ de rendimento, via o intermediário $\mathbf{8 3}$, que pode ser isolado controlando-se o potencial eletroquímico. Cumarinas são compostos de interesse farmacológico devido às suas potentes e variadas atividades. ${ }^{119,120}$

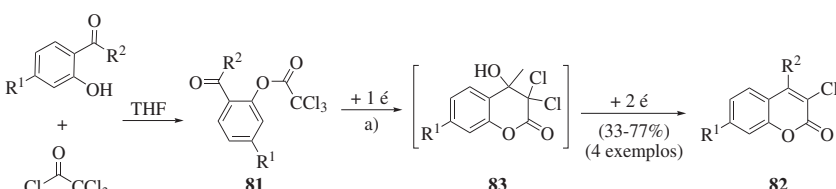

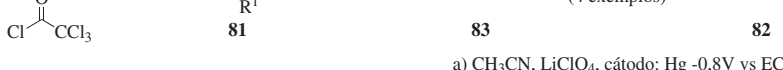

$$
\begin{aligned}
& \mathrm{R}^{1}=\mathrm{Me}, \mathrm{OMe} ; \mathrm{R}^{2}=\mathrm{H} \text {, alquil, fenil }
\end{aligned}
$$

\section{Esquema 27}

As reações de cetonas, ésteres e heterociclos contendo o grupo triclorometila com tióis, em presença de bases do tipo $\mathrm{NaH}, \mathrm{BuLi}$ ou $\mathrm{Et}_{3} \mathrm{~N}$, formam ditianos em vez de produtos de substituição de $-\mathrm{CCl}_{3} \cdot{ }^{28,70,121}$ Quando essas reações são realizadas com ditióis, formam-se ditianos heterociclos derivados de $\mathbf{8 4}$, com rendimentos entre 82 a $92 \%$ (Esquema 28). ${ }^{121}$ Ditianos, conhecidos por serem estáveis tanto em meio ácido quanto básico, são importantes para a formação de ligações carbono-carbono pelo emprego da abordagem da inversão de polaridade.

Uma classe de CCTH muito versátil para a síntese de heterociclos são os derivados de 1,1,1-tricloro-4-alcóxi-3-alquen-2-onas.

$$
\begin{aligned}
& \mathrm{R}-\mathrm{CCl}_{3}+\mathrm{HS} \overbrace{\mathrm{SH}} \mathrm{NaH,} \text { DMF, } 0{ }^{\circ} \mathrm{C}, 5-30 \mathrm{~min} \\
& (82-92 \%)
\end{aligned}
$$

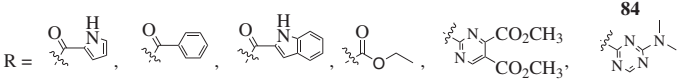

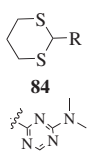

Esquema 28

No Esquema 29 está apresentada a reação da triclorocetona $\mathbf{8 5}$ com 2,3-diaminopiridina, em meio alcoólico básico, formando os heterociclos 86 com rendimentos de 54 a $70 \%$. ${ }^{122}$ Essas tricloroenonas são versáteis, servindo para a síntese de diversos heterociclos como pirazóis, isoxazóis, pirimidinas, benzimidazóis, inclusive heterociclos contendo o grupo $-\mathrm{CCl}_{3}{ }^{123}$ Esses heterociclos são conhecidos por suas inúmeras aplicações em Bioquímica ${ }^{124}$ e Química de materiais. ${ }^{125}$

$$
\begin{aligned}
& \text { Cly } 3 \text { C } \\
& 85 \\
& \begin{array}{c}
\mathbf{8 6} \\
\mathrm{R}=\text { aril, het. }
\end{array}
\end{aligned}
$$

Esquema 29

A HCA também atua como precursora para obtenção de heterociclos. As reações de HCA com diaminas, ditióis, sob energia de ultrassom, ${ }^{107-109}$ e com dióis, com diferentes catalisadores hidrossilicatos, ${ }^{126,127}$ levaram à obtenção de ureias, ditiocarbonatos e carbonatos, todos cíclicos, conforme exemplificado pelos compostos 87, 88 e 89, obtidos com 61,50 e $98 \%$ de rendimento, respectivamente (Esquema 30). Esses exemplos indicam que a HCA é um reagente alternativo ao tóxico fosgênio na preparação de vários heterociclos..$^{107,108}$

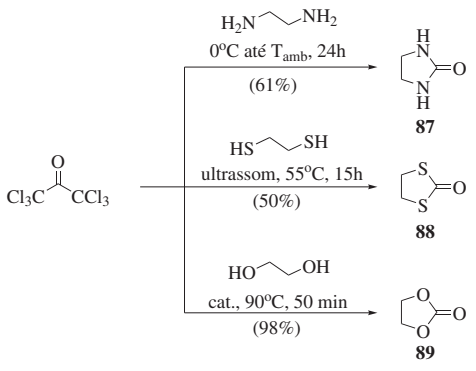

Esquema 30

A HCA reage com excesso (10 vezes) de diferentes di- e polinucleófilos para formar heterociclos "sem carbonila", sob energia de ultrassom, conforme exemplos apresentados no Esquema 31, relatados por Zucco e colaboradores. ${ }^{83-85,128-130}$ As reações de HCA com etilenodiamina e 1,2-diaminociclo-hexano, utilizando a própria diamina como solvente, produziram compostos 90 e 91, com rendimentos de 60 e 52\%, respectivamente. Já as reações de 1,2-diaminobenzeno

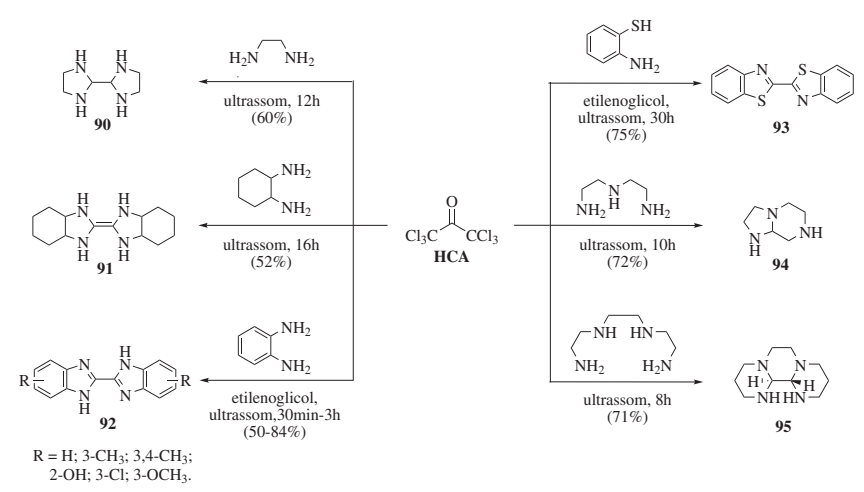

Esquema 31 
substituídos com 2-aminotiofenol, em etilenoglicol, propiciaram a obtenção dos derivados bis-benzimidazólicos 92 e de bis-benzotiazol 93, com rendimentos de 50-84\%. Por fim, as reações de HCA com dietilenotriamina e trietilenotetramina levaram aos triazabiciclo 94 e tetra-azatricíclico 95, respectivamente, rendendo 72 e $71 \%$. Ligantes heterociclos tais como os apresentados no Esquema 31, assim como seus complexos de coordenação, possuem uma enorme gama de aplicações em diferentes áreas. ${ }^{131-133}$

A HCA também é conhecida por catalisar a epoxidação de duplas ligações, especialmente em espécies esteroidais. ${ }^{134,135}$ Destaca-se, como exemplo, a reação com trietoxifosfito, para formar o fosfato enólico pentaclorado 96, com 50\% de rendimento, que reage via cicloadição [4+3] com furanos substituídos 97, em presença de uma solução de 2,2,2-trifluoretóxido de sódio em 2,2,2-trifluoretanol, para formar os oxabiciclos polissubstituídos 98, com rendimentos de 39 a $45 \%$ (Esquema 32). ${ }^{136}$ As reações com ciclopentadienos, nas mesmas condições, formaram os respectivos biciclos com rendimentos moderados.

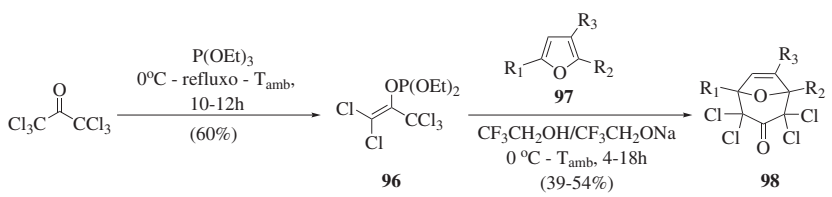

$\mathrm{R}_{1}=\mathrm{H}, \mathrm{CH}_{3} ; \mathrm{R}_{2}=\mathrm{H}, \mathrm{CH}_{3}, \mathrm{CH}_{2}=\mathrm{CHCH}_{2} \mathrm{CH}_{2}, \mathrm{CH}_{2}=\mathrm{C}\left(\mathrm{CH}_{3}\right) \mathrm{CH}_{2} \mathrm{CH}_{2} ; \mathrm{R}_{3}=\mathrm{H}, \mathrm{CH}_{3}$

\section{Esquema 32}

\section{HETEROCICLOS CONTENDO - $\mathrm{CX}_{3}$}

Heterociclos são usados nas várias áreas da Química, Bioquímica, Farmácia e Materiais. Os CCTHs são assumidos como precursores para a obtenção de heterociclos contendo a unidade $-\mathrm{CCl}_{3}$.

Zanatta, Martins, Bonacorso, Flores e colaboradores publicaram vários trabalhos de síntese de heterociclos contendo o grupo $-\mathrm{CCl}_{3} \mathrm{e}$ também avaliaram as atividades biológicas para diferentes fins. ${ }^{137-149}$ Foram utilizados por esse grupo, principalmente, os derivados de 4-alcoxi-1,1,1-tricloro-alquen-2-onas, que, conforme já mencionado, são reagentes de larga versatilidade. Mais recentemente, o mesmo grupo desenvolveu novas metodologias sintéticas utilizando energia de micro-ondas e ultrassom. O Esquema 33 mostra a síntese de dois ligantes não simétricos. A reação da triclorocetona $99 \mathrm{com}$ hidrato de hidrazina 6-hidrazino-nicotínica, 100, leva a 101, contendo duas unidades 3,4-di-hidro-3-hidroxi-3-triclorometil-pirazol e um grupo piridina, com rendimentos de 62 a 97\%, que é aromatizado ao derivado contendo duas unidades pirazoicas e uma piridínica 102. ${ }^{137}$ Esses ligantes, preparados por métodos bastante simples, têm aplicações na Química de coordenação. ${ }^{137}$ Em outro exemplo, a preparação de derivados isoxazólicos contendo o grupo triclorometila é realizada

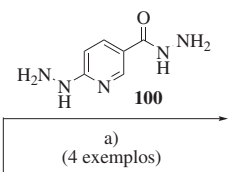

$$
\begin{aligned}
& \mathrm{MeO}_{\mathrm{CCl}_{3}}^{\mathrm{R}} \\
& 99
\end{aligned}
$$

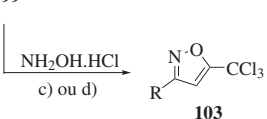

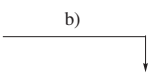

101

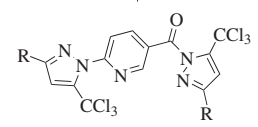

102

a) EtOH, refluxo, $4 \mathrm{~h} ; \mathrm{R}=$ aril $(62-85 \%)$;

b) $\mathrm{SOCl}_{2}$, piridina, benzeno, $0-80^{\circ} \mathrm{C}, \mathrm{R}=$ fenil $(64 \%)$;

c) $\mathrm{MeOH}$, piridina, microondas, $100 \mathrm{~W}, 70^{\circ} \mathrm{C}, 2,2 \mathrm{bar}, 6 \mathrm{~min}$, $\mathrm{H}_{2} \mathrm{SO}_{4}$ conc., microondas, $80{ }^{\circ} \mathrm{C}, 3,5$ bar, $\mathrm{R}=$ aril $(78-90 \%)$; d) $\mathrm{HCl} \mathrm{exc,} \mathrm{MeOH}, 70^{\circ} \mathrm{C}, 48 \mathrm{~h}, \mathrm{R}=$ aril $(77-85 \%)$. a partir de 99 com cloridrato de hidroxilamina, gerando os produtos derivados de 103, sob energia de micro-ondas ou condições térmicas. Com a utilização de micro-ondas foram obtidos melhores rendimentos em menores tempos de reações. ${ }^{138}$

Outros derivados heterociclos contendo $-\mathrm{CCl}_{3}$ incluem o composto pirimidínico 104, ${ }^{139}$ a pirididona $105,{ }^{123}$ os derivados de pirazol e isoxazol, representados por 106, ${ }^{140}$ e do selênio, 107 (Figura 2). ${ }^{123}$
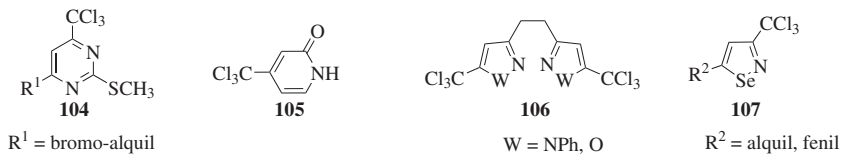

Figura 2. Exemplos de heterociclos contendo $-\mathrm{CCl}_{3}$ obtidos por Zanatta, Martins, Bonacorso, Flores e colaboradores

Yavari e colaboradores sintetizaram heterociclos substituídos a partir das reações de HCA com acetilenos ativados por dois grupos ésteres ou cetonas, conforme o Esquema 34. ${ }^{150,151}$ Nas reações de HCA com as dicetonas, com isocianeto, foram obtidos os derivados $\mathbf{1 0 8}$ com ótimos rendimentos (85 a 92\%). Nas reações de HCA com O-alquil diéteres, em presença de trifenoxifosfito, foram obtidos os heterociclos 109, contendo um grupo triclorometila além de um diclorovinila, com rendimentos de 65 a 95\%. Todavia, quando as reações são realizadas com $\mathrm{O}$-alquil ou $\mathrm{O}$-aril diésteres, em presença de isocianeto, foram obtidos os heterociclos 110, contendo dois grupos triclorometila, com 65 a $90 \%$ de rendimento. Esses compostos são importantes do ponto de vista biológico, podendo atuar como blocos de construção para obtenção de novos agentes em função de suas polifuncionalidades.

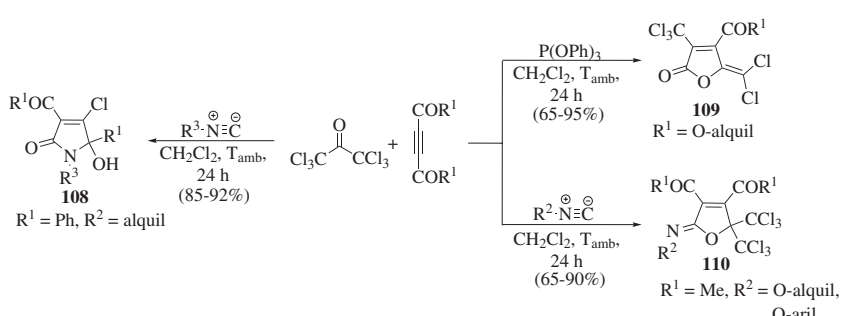

Esquema 34

\section{Aplicações sintéticas de heterociclos contendo - $\mathbf{C X}_{3}$}

Outros exemplos de heterociclos contendo o grupo $-\mathrm{CX}_{3}$, não necessariamente obtidos a partir de CCTH, são descritos na literatura. A reação de 2-metilquinoxalina, 111, com uma mistura de $\mathrm{POCl}_{3} /$ $\mathrm{PCl}_{5}$ sob energia de micro-ondas produz 2-(triclorometil)quinoxalina, 112, que reage com aldeídos aromáticos, em 1,1,2,2-tetra-dimetilaminoetano (TDAE), para formar $\alpha$-clorocetonas quinoxalínicas 113, com rendimentos de 27 a $69 \%$ (Esquema 35). ${ }^{152} \mathrm{O}$ mecanismo dessas reações ocorre via formação de uma espécie cloroxirano, geralmente instáveis, mas que pode ser isolado com $24 \%$ de rendimento (114), a partir da reação de 112 com 4-nitroacetofenona, nas mesmas condições experimentais. Por fim, uma adaptação do método para derivados de quinolina levou às $\alpha$-clorocetonas com rendimentos de $37-59 \% .{ }^{152}$

Um exemplo interessante de aplicação envolvendo derivados quinolínicos e isoquinolínicos diz respeito à expansão do anel, mediado por $\mathrm{AgNO}_{3}$, conforme descrito por Guingant e colaboradores, ${ }^{153,154}$ exemplificado no Esquema 36. Após ser protegido com brometo de metila, o sal isoquinolínico $\mathbf{1 1 5}$ reage com bromofórmio, em meio básico, produzindo o derivado tribromado 116 que, com $\mathrm{AgNO}_{3} \mathrm{em}$ metanol, via formação do íon dibromo-aziridínico 117 , sofre ataque 


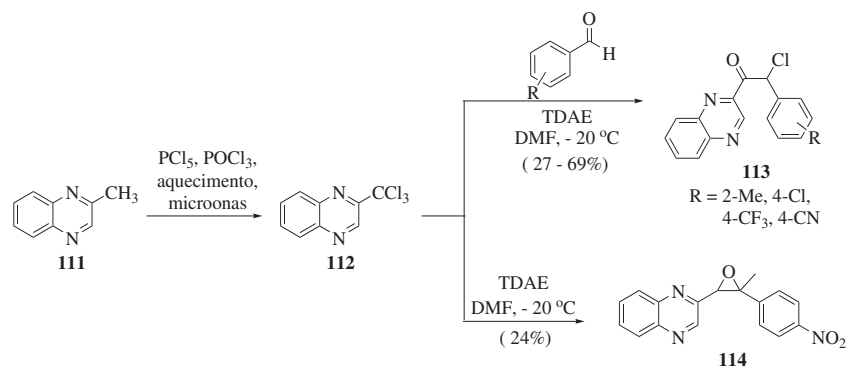

Esquema 35

nucleofílico do álcool culminando na espécie 118, por expansão para anel de 7 membros. A hidrólise dos grupos bromo, gerando carbonila, resulta no derivado 119. Esse método é utilizado tanto na preparação de $2 \mathrm{H}$-1- quanto de $2 \mathrm{H}$-3-benzapin-2-onas, compostos que possuem importantes aplicações na Química medicinal, como o antiangina Zatebradina, preparado com bom rendimento global. ${ }^{153,154}$

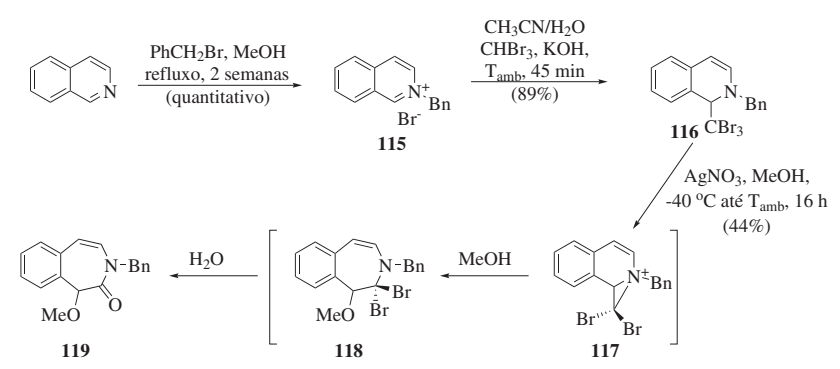

Esquema 36

$\mathrm{O}$ derivado imidazólico $\mathbf{1 2 0}$ contendo os grupos nitro e $-\mathrm{CCl}_{3}$ preparado a partir da reação de 2-metil-5-nitro-N-metilimidazol com $\mathrm{CCl}_{4}$, em meio básico, reage com 2-nitropropano, na presença de hidróxido de tetra-butilamônio (TBAOH) para formar o cloreto vinílico imidazólico $\mathbf{1 2 1}$, com rendimento de $76 \%$, por meio de mecanismo radicalar do tipo $\mathrm{S}_{\mathrm{RN}} 1$ e $\mathrm{R}_{\mathrm{RC}} 1$, consecutivo (Esquema 37). ${ }^{155}$

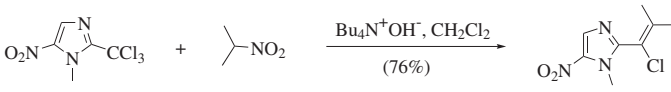

$$
\begin{aligned}
& 120
\end{aligned}
$$

\section{Esquema 37}

Yanai e Taguchi demonstraram que reações de tricloroacetaldeído $N, O$-acetais $\mathbf{1 2 2}$, obtidos a partir da reação de cloral com 2-aminofenóis- $N$-substituídos, quando em presença de $n$-BuLi levam à formação dos intermediários fenoxienaminas $\mathbf{1 2 3}$, que são protonados aos derivados diclorometilados $\mathbf{1 2 4}$, ou reagem com outros eletrófilos, como aldeídos, para formar os compostos $\mathbf{1 2 5}$ ou com trifluoretil- $\alpha$-cetoacetato de etila para formar 126 (Esquema 38). ${ }^{156}$ Os produtos dessas reações, importantes para Química de materiais e medicinal, ${ }^{157}$ foram obtidos com rendimentos variando de 51 a $94 \%$.

Outros exemplos de expansão de anel mediados por CCTH são os derivados pirrólicos triclorometilados em piridínicos clorados, ${ }^{157}$ substratos adequadamente ativados para reações de substituição nucleofílica. ${ }^{158,159}$ Ainda, as reações de cumarinas nitradas e trihalogenadas podem ser convertidas em derivados de pirazolidinas polissubstituídas. ${ }^{160}$

Recentemente, Fesenko e colaboradores publicaram uma nova metodologia para a síntese de 5-acil-1,2-di-hidropirimidin-2-onas, 127 (Esquema 39). ${ }^{161}$ Dessa forma, a reação de ureia com hidrato

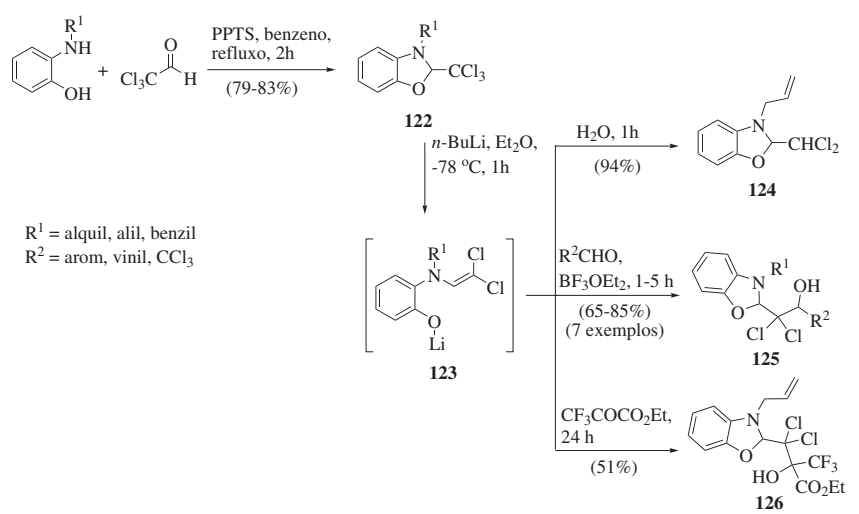

Esquema 38

de cloral leva à formação de $\mathbf{1 2 8}$, que pode ser mono- $O$ - ou duplamente - $O, N$-acilado para formar 129 e 130 , respectivamente. Tanto 129 quanto 130 reagem com enolatos formados a partir das reações dos compostos 1,3-dicarbonilados, 131, com NaH, para formar os derivados 132, via substituição nucleofílica do grupo -OAc. Esses compostos, quando controladas as condições experimentais, formam unicamente as 4-triclorometil-1,2,3,4-tetra-hidropirimidin-2-onas 133, com rendimentos de 75 a $95 \%$, como intermediários-chave para formação de 127 (que se encontra em equilíbrio com sua forma tautomérica 134), com rendimentos de 70 a $88 \%$, via eliminação de $\mathrm{CHCl}_{3}$ pelo tratamento com NaH. As di-hidropirimidin-2-onas são heterociclos de interesse por possuírem atividades biológicas. ${ }^{161}$

$$
\begin{aligned}
& \mathrm{R}^{1}=\mathrm{Me}, \mathrm{Ph} \\
& \mathrm{R}^{2}=\mathrm{Me}, \mathrm{Ph}, \mathrm{OEt}
\end{aligned}
$$

\section{Esquema 39}

O Esquema 40 apresenta a síntese de diaminas vicinais quirais derivadas de prolina, conhecidas como ligantes em uma variedade de reações enantiosseletivas. ${ }^{162}$ A reação de cloral com prolina, 135, ou oxo-prolina, 136, leva à obtenção dos heterociclos triclorometilados 137 e 138, respectivamente, que podem ser transformados nas amidas 139 e 140, por abertura nucleofílica do anel com aminas. As amidas $N$ formiladas 141 podem ser obtidas como intermediários na conversão de 137 em 138, controlando-se a quantidade de amina utilizada. Por
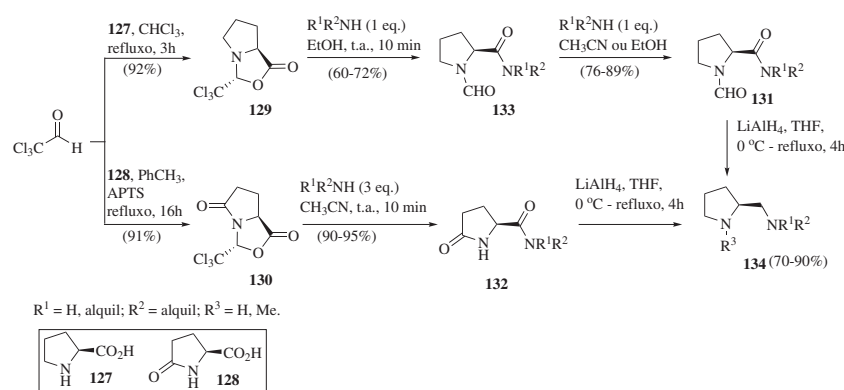

Esquema 40 
fim, as reduções de 139 e 140, com $\mathrm{LiAlH}_{4}$, possibilitam a obtenção das diaminas quirais $\mathbf{1 3 4}$, com 70 a $90 \%$ rendimento.

\section{CONSIDERAÇÕES FINAIS}

Compostos orgânicos contendo os grupos $-\mathrm{CCl}_{3} \mathrm{e}-\mathrm{CBr}_{3}$ possuem importantes aplicações em diferentes áreas, com destaque para seus usos como precursores sintéticos para várias funções químicas. Neste estudo, foram escolhidos exemplos relatados na literatura, especialmente na última década, cujas fontes estão disponíveis no Portal de Periódicos da CAPES, no Sciencedirect e no Scifinder. Ratificou-se, pela pesquisa, o inegável fato de que os derivados clorados são muito mais explorados que os derivados bromados.

Além de juntar informações acerca da utilização dos compostos contendo $-\mathrm{CCl}_{3} \mathrm{e}-\mathrm{CBr}_{3}$, a presente revisão tem como objetivo encorajar a busca por novas metodologias baseadas nesses compostos.

\section{REFERÊNCIAS}

1. D'Amato, C.; Torres, J. P. M.; Malm, O.; Quim. Nova 2002, 25, 995.

2. Tiwari, A.; Waud, W. R.; Struck, R. F.; Bioorg. Med. Chem. 2002, 10, 3593.

3. Liu, X.; Narla, R. K.; Uckun, F. M.; Bioorg. Med. Chem. Lett. 2003, 13, 581.

4. Boyce, M.; Bryant, K. F.; Jousse, C.; Long, K.; Harding, H. P.; Scheuner, D.; Kaufman, R. J.; Ma, D.; Coen, D. M.; Ron, D.; Yuan, J.; Science 2005, 307, 935.

5. Long, K.; Boyce, M.; Lin, H.; Juan, J.; Ma, D.; Bioorg. Med. Chem. Lett. 2005, 15, 3849.

6. Bringmann, G.; Feineis, D.; Brückner, R.; Blank, M.; Peters, K.; Peters, E.; Reichmann, H.; Janetzky, B.; Grote, C.; Clement, H.; Wesemann, W.; Bioorg. Med. Chem. 2000, 8, 1467.

7. Sugano, M.; Sato, A.; Nagaki, H.; Yoshioka, S.; Shiraki, T.; Horikoshi, H.; Tetrahedron Lett. 1990, 31, 7015.

8. Fuson, R. C.; Bull, B. A.; Chem. Rev. 1934, 15, 275.

9. Sahu, B.; Gururaja, G. N.; Mobin, S. M.; Namboothiri, I. N. N.; J. Org. Chem. 2009, 74, 2601.

10. Concellón, J. M.; Bernard, P. L.; Méjica, C.; Tetrahedron Lett. 2005, 46, 569.

11. Chinoporos, E.; Chem. Rev. 1963, 63, 235.

12. Gukasyan, A.O.; Galstyan, L.K.; Avetisyan, A.A. Russ. Chem. Rev. 1991, 60, 1318.

13. Corey, E. J.; Link, J. O.; J. Am. Chem. Soc. 1992, 114, 1906.

14. Corey, E. J.; Link, J. O.; Shao, Y.; Tetrahedron Lett. 1992, 8, 5881.

15. Mellin-Morlière, C.; Aitken, D. J.; Bull, S. D.; Davies, S. G.; Husson, H.; Tetrahedron: Asymmetry 2001, 12, 149.

16. Baati, R.; Barma, D. K.; Falck, J. R.; Mioskowski, C.; Tetrahedron Lett. 2002, 43, 2183.

17. Aggarwal, V. K.; Mereu, A.; J. Org. Chem. 2000, 65, 7211.

18. Robertson, J.; Fowler, T. G.; Org. Biomol. Chem. 2006, 4, 4307.

19. Katosh, M.; Hisa, C.; Honda, T.; Tetrahedron Lett. 2007, 48, 4691.

20. Dominguez, C.; Ezquerra, J.; Baker, S. R.; Borrelhy, S.; Prieto, L.; Espada, M.; Pedregal, C.; Tetrahedron Lett. 1998, 39, 9305.

21. Zhu, J.; Blanchet, J.; Tetrahedron Lett. 2004, 45, 4449.

22. Habay, S. A.; Schefmeister, C. E.; Org. Lett. 2004, 6, 3369.

23. Sørensen, M. H.; Nielsen, C.; Nielsen, P.; J. Org. Chem. 2001, 66, 4878.

24. Corey, E. J.; Helal, C.; Angew. Chem., Int. Ed. 1998, 37, 1986.

25. Cafiero, L. R.; Snowden, T. S.; Org. Lett. 2008, 10, 3853.

26. Wang, Z.; Campagna, S.; Yang, K.; Xu, G.; Pierce, M. E.; Fortunak, J. M.; Confalone, P. N.; J. Org. Chem. 2000, 65, 1889.

27. Cafiero, L. R.; Snowden, T. S.; Tetrahedron Lett. 2008, 49, 2844.

28. Morimoto, H.; Lu, G.; Aoyama, N.; Matsunaga, S.; Shibasaki, M.; J. Am. Chem. Soc. 2007, 129, 9588.
29. Corey, E. J.; Helal, C. J.; Angew. Chem., Int. Ed. 1998, 37, 1986.

30. Kabalka, G. W.; Wu, Z.; Ju, Y.; Yao, M.; J. Org. Chem. 2005, 70, 10285.

31. Si, Y.; Guo, S.; Wang, W.; Jiang, B.; J. Org. Chem. 2005, 70, 1494.

32. Guiraldo, A.; Martiz, B.; Andreu, R.; Tetrahedron Lett. 2004, 45, 8523.

33. Guirado, A.; Martiz, B.; Andreu, R.; Bautista, D.; Gálvez, J.; Tetrahedron 2007, 63, 1175 .

34. Shevchenko, N. E.; Röschenthaler, G.; Mitiaev, A. S.; Lork, E.; Nenajdenko, V. G.; J. Fluorine Chem. 2008, 129, 637.

35. Ramachandran, P. V.; Gong, B.; Teodorović, A. V.; J. Fluorine Chem. 2007, 128, 844

36. Jocic, Z. Z.; Zh. Russ. Fiz. Khim. Ova. 1897, 29, 97 (retirado de: Oliver, J. E.; Schimidt, W.; Tetrahedron: Asymmetry 1998, 9, 1723).

37. Shamshina, J. L.; Snowden, T. S.; Org. Lett. 2006, 8, 5881.

38. Li, J.; Xu, X.; Zhang, Y.; Tetrahedron Lett. 2003, 44, 9349.

39. Ranu, B. C.; Samanta, S.; Das, A.; Tetrahedron Lett. 2002, 43, 5993.

40. D'Anna, F.; Frenna, V.; Pace, V.; Noto, R.; Tetrahedron 2006, 62, 1690.

41. Baati, R.; Barma, D. K.; Falck, J. R.; Miokoswski, C.; Tetrahedron Lett. 2002, 43, 2183.

42. Bejot, R.; Tisserand, S.; Reddy, L. M.; Barma, D. K.; Baati, R.; Falck, J. R.; Mioskowski, C.; Angew. Chem., Int. Ed. 2005, 44, 2008.

43. Ram, R. N.; Manoj, T. P.; Org. Lett. 2008, 10, 2243.

44. Sonogashira, K.; Tohda, Y.; Hagihara, N.; Tetrahedron. Lett. 1975, 50, 4467.

45. Sonogashira, K.; J. Organomet. Chem. 2002, 653, 46.

46. Gallardo, H.; Cristiano, R.; Vieira, A. A.; Neves Filho, R. A. W.; Srivastava, R. M.; Synthesis 2008, X, 605.

47. Merlo, A. A.; Braun, J. E.; Vasconcelo, U.; Ely, F.; Gallardo, H.; Liq. Cryst. 2000, 27, 657.

48. Vasconcelos, U. B.; Schrader, A.; Vilela, G. D.; Borges, A. C. A.; Merlo, A. A.; Tetrahedron 2008, 64, 4619.

49. Vögtle, F.; Sieger, H.; Müller, W. M.; J. Chem. Res. 1978, 398.

50. Cristiano, R.; Ely, F.; Gallardo, H.; Liq. Cryst. 2005, 32, 15.

51. Cristiano, R.; Westphal, E.; Bechtold, I. H.; Bortoluzzi, A. J.; Gallardo, H.; Tetrahedron 2007, 63, 2851.

52. Westphal, E.; Bechtold, I. H.; Gallardo, H.; Macromolecules 2010, 43, 1319.

53. van Esch, J. H.; Feringa, B. L.; Angew. Chem., Int. Ed. 2000, 39, 2263.

54. Vieira, A. A.; Cristiano, R.; Bortoluzzi, A. J.; Gallardo, H.; J. Mol. Struct. 2008, 875, 364.

55. Cristiano, R.; Gallardo, H.; Bortoluzzi, A. J.; Bechtold, I. H.; Campos, C. E. M.; Longo, R. L.; Chem. Commun. 2008, 41, 5134.

56. Velazquez, S.; Alvarez, R.; Perez, C.; Gago, F.; De J. Balzarini, C.; Camaraza, M.; Antiviral Chem. Chemother. 1998, 9, 481.

57. Rostovtsev, V. V.; Green, L. K.; Fokin, V. V.; Sharpless, K. B.; Angew. Chem., Int. Ed. 2002, 41, 2596.

58. Tornøe, C. W.; Christensen, C.; Meldal, M.; J. Org. Chem. 2002, 67, 3057.

59. Wang, Q.; Chen, T. R.; Hilgraf, R.; Fokin, V. V.; Sharpless, K. B.; Finn, M. G.; J. Am. Chem. Soc. 2003, 125, 3192.

60. Gallardo, H.; Ely, F.; Bortoluzzi, A. J.; Liq. Cryst. 2005, 32, 667.

61. Conte, G.; Ely, F.; Gallardo, H.; Liq. Cryst. 2005, 32, 1213.

62. Gallardo, H.; Conte, G.; Bryk, F.; Lourenço, M. C. S.; Costa, M. S.; Ferreira, V. F.; J. Braz. Chem. Soc. 2007, 18, 1285.

63. Cristiano, R.; Santos, D. M. P. D.; Conte, G.; Gallardo, H.; Liq. Cryst. 2006, 33, 997.

64. Conte, G.; Cristiano, R.; Ely, F.; Gallardo, H.; Synth. Commun. 2006, $36,951$.

65. Falck, J. R.; He, A.; Reddy, L. M.; Kundu, A.; Barma, D. K.; Bandyophdhyay, A.; Kamila, S.; Akella, R.; Bejot, R.; Mioskowski, C.; Org. Lett. 2006, $8,4645$.

66. Ram, N. R.; Manoj, T. P.; J. Org. Chem. 2008, 73, 5633.

67. Ram, R. N.; Kumar, N.; Tetrahedron Lett. 2008, 49, 799. 
68. Zucco, C.; Lima, C. F.; Rezende, M. C.; Vianna, J. F.; Nome, F.; J. Org. Chem. 1987, 52, 5356

69. Albanese, D.; Landini, D.; Penso, M.; J. Org. Chem. 1992, 57, 1603.

70. Hess, S. C.; Nome, F.; Zucco, C.; Rezende, M. C.; Synth. Commun. 1989, 19, 3037.

71. Salim, J. R.; Zucco, C.; Nome, F.; Quim. Nova 1991, 14, 244.

72. Uieara, M.; Zucco, C.; Zanette, D.; Rezende, M. C.; Nome, F.; J. Chem. Soc., Perkin Trans. 2 1987, X, 175.

73. Zanatta, N.; Faoro, D.; Silva, S. C.; Bonacorso, H. G.; Martins, M. A. P.; Tetrahedron Lett. 2004, 45, 5689.

74. Pettigrew, J. D.; Wilson, P. D.; Org. Lett. 2006, 8, 1427.

75. Bennasar, M.; Roca, T.; Zulaica, E.; Monerris, M.; Tetrahedron 2004, 60, 6785.

76. Rebelo, R. A.; Rezende, M. C.; Nome, F.; Zucco, C.; Synth. Commun. 1987, 17, 1741.

77. Salim, J. R.; Nome, F.; Rezende, M. C. ; Synth. Commun. 1989, 19, 1181.

78. Druzian, J.; Zucco, C.; Rezende, M. C.; Nome, F.; J. Org. Chem. 1989, $54,4767$.

79. Tiberghien, A. C.; Evans, D. A.; Kiakos, K.; Martin, C. R. H.; Hartley, J. A.; Thurston, D. E.; Howard, P. W.; Bioorg. Med. Chem. Lett. 2008, 18, 2073.

80. Han, X.; Li, C.; Mosher, M. D.; Rider, K. C.; Zhou, P.; Crawford, R. L.; Fusco, W.; Paszczynski, A.; Natale, N. R.; Bioorg. Med. Chem. 2009, 17, 1671.

81. Freedlander, R. S.; Bryson, T. A.; Dunlap, R. B.; Schulman, E. M.; Lewis, C. A.; J. Org. Chem. 1981, 46, 3520.

82. Menezes, F. G.; Kolling, R.; Bortoluzzi, A. J.; Gallardo, H.; Zucco, C.; Tetrahedron Lett. 2009, 50, 2559.

83. Rezende, M. C.; Marques, C. A.; Dall'Oglio, E. L.; Zucco, C.; Liebigs Ann., Recueil. 1997, 5, 925.

84. Dall'Oglio, E. L.; Tese de Doutorado, Universidade Federal de Santa Catarina, Brasil, 2002.

85. Rezende, M. C.; Dall'Oglio, E. L.; Zucco, C.; Tetrahedron Lett. 1996, 37,5265 .

86. Sonovskikh, V. Y.; Mel'nikov, M. Y.; Mendeleev. Commun. 1998, 8, 243.

87. Dolzhenko, A. V.; Patorin, G.; Dolzhenko, A. V.; Chi, W. C.; Tetrahedron Lett. 2008, 49, 7180

88. Nishikawa, T.; Urabe, D.; Tomita, M.; Tsujimoto, T.; Iwabuchi, T.; Isobe, M.; Org. Lett. 2006, 8, 3263.

89. Mitsunobu, O.; Yamada, Y.; Bull. Chem. Soc. Jpn. 1967, 40, 2380.

90. Clayden, J.; Greeves, N.; Warren, S.; Wothers, P.; Organic Chemistry, $1^{\text {st }}$ ed.; Oxford: New York, 2001.

91. Pluempanupat, W.; Chantarasriwong, O.; Taboonpong, P.; Jang, D. O.; Chavasiri, W.; Tetrahedron Lett. 2007, 47, 223.

92. Kang, D. O.; Joo, T. Y.; Lee, E. H.; Chaysripongkul, S.; Chavasiri, W.; Jang, D. O.; Tetrahedron Lett. 2006, 47, 5693.

93. Pluempanupat, W.; Chavasiri, W.; Tetrahedron Lett. 2006, 47, 6821.

94. Tongkate, P.; Pluempanupat, W.; Chavasiri, W.; Tetrahedron Lett. 2008, $49,1146$.

95. George, T. G.; Endeshaw, M. M.; Morgan, R. E.; Mahasenan, K. V.; Delfín, D. A.; Mukherjee, M. S.; Yakovich, A. J.; Fotie, J.; Li, C.; Werbovetz, K. A.; Bioorg. Med. Chem. 2007, 15, 6071.

96. Lovchik, M. A.; Goeke, A.; Fráter, G.; J. Org. Chem. 2007, 72, 2427.

97. Bergin, E.; O'Connor, C. T.; Robinson, S. B.; McGarrigle, E. M.; O'Mahony, C. P.; Gilheany, D.; J. Am. Chem. Soc. 2007, 129, 9566.

98. Stankevič, M.; Pietrusiewicz, K. K.; J. Org. Chem. 2007, 72, 816.

99. Wang, B.; Yu, C. M.; Chen, Z. W.; Su, W. K.; Chin. Chem. Lett. 2008, 19, 904.

100. Laskovics, F. M.; Schulman, E. M.; J. Am. Chem. Soc. 1977, 99, 6672.

101. Meredith, W.; Nemeth, G. A.; Boucher, R.; Carney, R.; Haas, M.; Sigvardson, K.; Teleha, C. A.; Tetrahedron Lett. 2003, 44, 7381.
102. Kumar, M.; Pandey, S. K.; Gandhi, S.; Singh, V. K.; Tetrahedron Lett. 2009, 50, 363.

103. Bisol, T. B.; Sá, M. M.; Quim. Nova 2007, 30, 106.

104. Majundar, K. C.; Alam, S.; Chattopadhyay, B.; Tetrahedron 2008, 64, 597.

105. Hama, N.; Matsuda, T.; Sato, T.; Chida, N.; Org. Lett. 2009, 11, 2687.

106. Swift, M. D.; Sutherland, A.; Tetrahedron 2008, 64, 9521.

107. Zaed, A. M.; Swift, M. D.; Sutherland, A.; Org. Biomol. Chem. 2009, 7 , 2678.

108. Swift, M. D.; Donaldson, A.; Sutherland, A.; Tetrahedron Lett. 2009, 50, 3241.

109. Barma, D. K.; Hundu, A.; Zhang, H.; Mioslowski, C.; Falck, J. R.; J. Am. Chem. Soc. 2003, 125, 3218.

110. Barma, D. K.; Lu, B.; Baati, R.; Mioskowski, C.; Falck, J. R.; Tetrahedron Lett. 2008, 49, 4359.

111. Falck, J. R.; Bandyopadhyay, A.; Barma, D. K.; Shin, D.; Kundu, A.; Kishore, V. R.; Tetrahedron Lett. 2004, 45, 3039.

112. Falck, J. R.; Bejot, R.; Barma, D. K.; Bandyopadhyay, A.; Joseph, S.; Mioskowski, C.; J. Org. Chem. 2006, 71, 8178.

113. Concellón, J. M.; Rodríguez-Solla, H.; Diaz, P.; Org. Biomol. Chem. 2008, 6, 2934.

114. Concellón, J. M.; Rodríguez-Solla, H.; Diaz, P.; Org. Biomol. Chem. 2008, 6, 451.

115. Shen, Y.; Zhang, Y.; Org. Lett. 2001, 3, 2805.

116. Seigal, B. A.; Fajardo, C.; Snapper, M. L.; J. Am. Chem. Soc. 2005, 127, 16329.

117. Ishibashi, H.; Sasaki, M.; Taniguchi, T.; Tetrahedron 2008, 64, 7771.

118. Lee, B. T.; Schrader, T. O.; Martín-Matute, B.; Kauffman, C. R.; Zhang, P.; Snapper, M. L.; Tetrahedron 2004, 60, 7391.

119. Batanero, B.; Barba, F.; Electrochem. Commun. 2001, 3, 595.

120. Batanero, B.; Pérez, M. J.; Barba, F.; J. Electroanal. Chem. 1999, 469, 201.

121. Rivera, N. G.; Becerril, D. C.; Guadarrama-Perez, C.; CovarrubiasZuñiga, A.; Avila-Zárraga, J. G.; Romero-Ortega, M.; Tetrahedron Lett. 2007, 48, 1201

122. Bonacorso, H. G.; Lourega, R. V.; Deon, E. D.; Zanatta, N.; Martins, M. A. P.; Tetrahedron Lett. 2007, 48, 4835.

123. Martins, M. A. P.; Cunico, W.; Pereira, C. M. P.; Sinhorin, A. P.; Flores, A. F. C.; Bonacorso, H. G.; Zanatta, N.; Curr. Org. Synth. 2004, 1, 391.

124. Romeiro, G. A.; Pereira, L. O. R.; de Souza, M. C. B. V.; Ferreira, V. F.; Cunha, A. C.; Tetrahedron Lett. 1997, 38, 5103.

125. Cristiano, R.; Gallardo, H.; Bortoluzzi, A. J.; Bechtold, I. H.; Campos, C. E. M.; Longo, R. L.; Chem. Commun. 2008, 41, 5134.

126. Gordon, E. P.; Elesina, L. N.; Enakaeva, V. G.; Mitrokhin, A. M.; Poddubnyi, I. S.; RU pat. 23099352007 (CAN 147:522096).

127. Gordon, E. P.; Elesina, L. N.; Enakaeva, V. G.; Mitrokhin, A. M.; Poddubnyi, I. S.; RU pat. 23099342007 (CAN 147:504235).

128. Rezende, M. C.; Dall'Oglio, E. L.; Zucco, C.; Synth. Commun. 2001, $31,607$.

129. Dall'Oglio, E. L.; Caro, M. S. B.; Gesser, J. C.; Zucco, C.; Rezende, M. C.; J. Braz. Chem. Soc. 2002, 13, 251.

130. Zucco, C.; Dall'Oglio, E. L.; Salmoria, G. V.; Gallardo, H.; Neves, A.; Rezende, M. C.; J. Phys. Org. Chem. 1998, 11, 411.

131. Miranda, F. S.; Menezes, F. G.; Vicente, J.; Bortoluzzi, A. J.; Zucco, C.; Neves, A.; Gonçalves, N. S.; J. Mol. Struct. 2007, 938, 1.

132. Meyer, E.; Zucco, C.; Gallardo, H.; J. Mater. Chem. 1998, 8, 1351.

133. Gallardo, H.; Meyer, E.; Bortoluzzi, A. J.; Molin, F.; Mangrich, A. S.; Inorg. Chim. Acta 2004, 357, 505.

134. Larkin, J. P.; Wehrey, C.; Boffelli, P.; Lagraulet, H.; Lemaitre, G.; Medelec, A.; Prat, D.; Org. Process Res. Dev. 2002, 6, 20.

135. Francsics-Czinege, E.; Tube, Z.; Molnár, C.; Horváth, J.; Csörgei, J.; Visky, G.; Balogh, G.; Mák, M.; Hegedũs, B.; Magyari, M.; Horváth, J.; Steroids 2003, 68, 739 . 
136. Föhlisch, B.; Reiner, S.; Molecules 2004, 9, 1.

137. Bonacorso, H. G.; Paim, G. R.; Guerra, C. Z.; Sehnem, R. C.; Cechinel, C. A.; Porte, L. M. F.; Martins, M. A. P.; Zanatta, N.; J. Braz. Chem. Soc. 2009, 20, 509.

138. Martins, M. A. P.; Machado, P.; Piovesan, L. A.; Flores, A. F. C.; de Campos, M. M. A.; Scheidt, C.; Bonacorso, H. G.; Zanatta, N.; Monatsh. Chem. 2008, 139, 985.

139. Zanatta, N.; Flores, D. C.; Madruga, C. C.; Flores, A. F. C.; Bonacorso, H. G.; Martins, M. A. P.; Tetrahedron Lett. 2006, 47, 573.

140. Martins, M. A. P.; Cunico, W.; Siqueira, G. M.; Leidens, V. L.; Zanatta, N.; Bonacorso, H. G.; Flores, A. F. C.; J. Braz. Chem. Soc. 2005, 16, 275.

141. Bonacorso, H. G.; Oliveira, M. R.; Costa, M. B.; Drekener, R. L.; da Silva, L. B.; Zanatta, N.; Martins, M. A. P.; Heteroat. Chem. 2006, 17, 685.

142. Martins, M. A. P.; Brondani, S.; Leidens, V. L.; Flores, D. C.; Moura, S.; Zanatta, N.; Hörner, M.; Flores, A. F. C.; Can. J. Chem. 2005, 83, 1171.

143. Zanatta, N.; Faoro, D.; Fernandes, L. S.; Brondani, P. B.; Flores, D. C.; Flores, A. F. C.; Bonacorso, H. G.; Martins, M. A. P.; Eur. J. Org. Chem. 2008, 5832 .

144. Martins, M. A. P.; Moreira, D. N.; Frizzo, C. P.; Longhi, K.; Zanatta, N.; Bonacorso, H. G.; J. Braz. Chem. Soc. 2008, 19, 1368.

145. Martins, M. A. P.; Guarda, E. A.; Frizzo, C. P.; Marzari, M. R. B.; Moreira, D. N.; Zanatta, N.; Bonacorso, H. G.; Monatsh. Chem. 2008, 139, 1321.

146. Martins, M. A. P.; Machado, P.; Rosa, F. A.; Cunico, W.; Bonacorso, H. G.; Zanatta, N.; Mini-Rev. Org. Chem. 2008, 5, 53.

147. Martins, M. A. P.; Pereira, C. M. P.; Cunico, W.; Moura, S.; Rosa, F. A.; Peres, R. L.; Machado, P.; Zanatta, N.; Bonacorso, H. G.; Ultrason. Sonochem. 2006, 13, 364 .
148. Martins, M. A. P.; Peres, R. L.; Fiss, G. F.; Dimer, F. A.; Mayer, R.; Frizzo, C. P.; Marzari, M. R. B.; Zanatta, N.; Bonacorso, H. G.; J. Braz. Chem. Soc. 2007, 18, 1486.

149. Moreira, D. N.; Frizzo, C. P.; Longhi, K.; Zanatta, N.; Bonacorso, H. G.; Martins, M. A. P.; Monatsh. Chem. 2008, 139, 1049.

150. Yavari, I.; Sabbaghan, M.; Hossaini, Z.; Ghazanfarpour-Darjani, M.; Helv. Chim. Acta 2008, 91, 1144.

151. Yavari, I.; Sabbaghan, M.; Hossaini, Z.; Monatsh. Chem. 2008, 139, 625. 152. Montana, M.; Terme, T.; Vanelle, P.; Tetrahedron Lett. 2006, 47, 6573.

153. Jean-Gérad, L.; Pauver, M.; Collet, S.; Guingant, A.; Evain, M.; Tetrahedron 2007, 63, 11250.

154. Pauvert, M.; Collet, S.; Guingant, A. ; Tetrahedron Lett. 2003, 44, 4203.

155. Verhaeghe, P.; Rathelot, P.; Gellis, R. S.; Vanelle, P. ; Tetrahedron 2006, 62,8173

156. Yanai, H.; Taguchi, T. ; Tetrahedron 2010, 66, 4530.

157. Raheem, I. T.; Thiara, P. S.; Jacobsen, E. N.; Org. Lett. 2008, 10, 1577.

158. Lister, T.; Prager, R. H.; Tsaconas, M.; Wikinson, K. L.; Aust. J. Chem. 2003, 56, 913 .

159. Menezes, F. G.; Ricardo, J.; Dias, R.; Bortoluzzi, A. J.; Zucco, C.; Quim. Nova 2007, 30, 356.

160. Korotaev, V. Y.; Kutyashev, I. B.; Sosnovskikh, V. Y.; Kodess, M. I.; Mendeleev Commun. 2007, 17, 52.

161. Fesenko, A. A.; Solovyev, P. A.; Shutalev, A. D.; Tetrahedron 2010, 66, 940.

162. Amedjkouh, M.; Ahlberg, P.; Tetrahedron: Asymmetry 2002, 13, 2229. 